ARTICLE

\title{
A glycoprotein B-neutralizing antibody structure at $2.8 \AA$ uncovers a critical domain for herpesvirus fusion initiation
}

\author{
Stefan L. Oliver (10 ${ }^{1 \times}$, Yi Xing ${ }^{2,8}$, Dong-Hua Chen (1) ${ }^{3}$, Soung Hun Roh ${ }^{4}$, Grigore D. Pintilie ${ }^{5}$, \\ David A. Bushnell (1) ${ }^{3}$, Marvin H. Sommer ${ }^{1}$, Edward Yang ${ }^{1}$, Andrea Carfi ${ }^{2,9}$, Wah Chiu (1) $5,6,7,10$ \& \\ Ann M. Arvin (1) 1,6,10
}

Members of the Herpesviridae, including the medically important alphaherpesvirus varicellazoster virus (VZV), induce fusion of the virion envelope with cell membranes during entry, and between cells to form polykaryocytes in infected tissues. The conserved glycoproteins, $\mathrm{gB}, \mathrm{gH}$ and $\mathrm{gL}$, are the core functional proteins of the herpesvirus fusion complex. $g B$ serves as the primary fusogen via its fusion loops, but functions for the remaining $g B$ domains remain unexplained. As a pathway for biological discovery of domain function, our approach used structure-based analysis of the viral fusogen together with a neutralizing antibody. We report here a $2.8 \AA$ cryogenic-electron microscopy structure of native $g B$ recovered from VZV-infected cells, in complex with a human monoclonal antibody, 93k. This high-resolution structure guided targeted mutagenesis at the gB-93k interface, providing compelling evidence that a domain spatially distant from the gB fusion loops is critical for herpesvirus fusion, revealing a potential new target for antiviral therapies.

\footnotetext{
${ }^{1}$ Department of Pediatrics, Stanford University School of Medicine, Stanford, CA 94305, USA. ${ }^{2}$ GSK Vaccines, Cambridge, MA 02139, USA. ${ }^{3}$ Structural Biology, Stanford University School of Medicine, Stanford, CA 94305, USA. ${ }^{4}$ Department of Biological Sciences, Institute of Molecular Biology \& Genetics, Seoul National University, Seoul 08826, Korea. ${ }^{5}$ Bioengineering, Stanford University School of Medicine, Stanford, CA 94305, USA. ${ }^{6}$ Microbiology and Immunology, Stanford University School of Medicine, Stanford, CA 94305, USA. ${ }^{7}$ Division of Cryo-EM and Bioimaging SSRL, SLAC National Accelerator Laboratory, Menlo Park, CA 94025, USA. ${ }^{8}$ Present address: Syros Pharmaceuticals, Inc. Cambridge, MA 02140, USA. ${ }^{9}$ Present address: Moderna,

Cambridge, MA 02139, USA. ${ }^{10}$ These authors jointly supervised this work: Wah Chiu, Ann M. Arvin. ${ }^{{ }}$email: sloliver@stanford.edu
} 
$\mathrm{M}$ embers of the Herpesviridae are pathogens of humans and animals that cause a wide range of medically and economically important diseases ${ }^{1}$. Herpesvirus virions have an outer lipid membrane studded with glycoproteins that enable fusion with cell membranes to initiate entry and establish infection. The most conserved herpesvirus protein, glycoprotein $\mathrm{B}$ $(\mathrm{gB})$, is classified as a type III fusogen due to its structural similarities with vesicular stomatitis virus (VSV) G protein and baculovirus gp $64^{2-8}$. In contrast to these fusogens, gB requires additional virally encoded glycoproteins, $\mathrm{gH}$ and gL, which are implicated in membrane binding and priming of the $\mathrm{gB}$ trimer, to induce membrane fusion ${ }^{9}$. Together, $\mathrm{gB} / \mathrm{gH}$-gL form the core herpesvirus fusion complex. Herpesvirus $\mathrm{gB}$ has been predicted to transition from a prefusion to a postfusion state ${ }^{2-7}$, but the molecular dynamics of the fusion complex is not understood.

Structures of the gB ectodomains of herpes simplex virus 1 (HSV-1), HSV-2, pseudorabies virus (PRV), human cytomegalovirus (HCMV), and Epstein Barr virus (EBV), determined by Xray crystallography, have identified five domains (I to V) that are similar to those of VSV G protein ${ }^{2-6,10,11}$. The C-terminal domain (CTD) structure of the HSV-1 gB was obtained by X-ray crystallography at $3.6 \AA$ resolution ${ }^{12}$. However, only lowresolution structures $(>24 \AA$ ) of herpesvirus $\mathrm{gB}$ in a putative prefusion form have been identified on exosomes derived from HSV-1 gB transfected cells and on HCMV particles using cryogenic electron tomography (cryo-ET) ${ }^{10,13}$. This has limited the ability to accurately model prefusion conformations of $\mathrm{gB}$.

Monoclonal antibodies (mAbs) to herpesvirus $\mathrm{gB}$ orthologues that neutralize viral infection are important for mapping functional domains because their activity depends on binding to $\mathrm{gB}$ before membrane fusion ${ }^{4,14-19}$. Antibodies that bind to DI, DII, and DIV of $\mathrm{gB}$ orthologues have neutralizing activity against several herpesviruses $4,15,20-23$. Although the molecular interactions for some of these antibodies with $\mathrm{gB}$ residues have been defined, it is currently not known whether these gB residues have roles in fusion function or virus infection.

Varicella-zoster virus (VZV) is a highly infectious, human host restricted alphaherpesvirus that causes varicella (chickenpox), establishes latency in sensory ganglion neurons and can reactivate to manifest as zoster (shingles) ${ }^{24}$. In addition to virion entry fusion, cell-cell fusion (abbreviated as cell fusion) is fundamental for VZV pathogenesis. Characteristic polykaryocytes form within tissues in vivo and are modeled in vitro by syncytia formation during MeWo cell infection ${ }^{25,26}$. Critically, adverse health effects are directly linked to the capability of VZV to overcome the usual constraint against fusion between differentiated host cells, causing fusion of ganglion neurons and satellite cells associated with postherpetic neuralgia (PHN), and fusion of vascular endothelial cells (giant cell arteritis) linked to strokes ${ }^{27-29}$.

VZV gB, a 931 amino acid (aa) protein encoded by open reading frame (ORF) 31, together with the VZV gH-gL heterodimer, trigger cell fusion in vitro, in the absence of other viral proteins ${ }^{30-32}$. The purpose of this study was to use the neutralizing human mAb $93 \mathrm{k}$ as a pathway to biological discovery of $\mathrm{gB}$ functional domains. A 2.8-Å resolution cryo-EM structure of native, full-length VZV gB in complex with $\mathrm{mAb}$ 93k Fab fragments was determined, revealing residues within gB DIV that were then shown to be essential for membrane fusion by evaluating DIV mutants in a virus-free assay. Mutagenesis of the VZV genome demonstrated their significance for $\mathrm{gB}$ fusion functions necessary to produce infectious extracellular VZV virions and for cell fusion to form syncytia. These findings have implications for modeling the transition of $\mathrm{gB}$ from prefusion to postfusion conformations. This study is highly relevant for developing novel therapies that inhibit infection by disrupting $\mathrm{gB}$ DIV-dependent molecular mechanisms of cell entry or cell fusion by members of the Herpesviridae.

\section{Results}

Human mAb 93k neutralizes VZV and inhibits fusion. The biological activity of mAb 93k or its Fab fragments was demonstrated by neutralization of a cell-free inoculum of the VZV strain, pOka (Fig. 1a). Importantly, 93k also reduced gB/gH-gLmediated membrane fusion by $95 \%$ in the stable reporter fusion assay (SRFA) in the absence of other viral proteins (Fig. 1b). Similar to $\mathrm{mAb} 93 \mathrm{k}$, the anti $\mathrm{gB}$ mAb SG2 ${ }^{33}$ bound to full-length $\mathrm{gB}$ that was purified from infected MeWo cells (Fig. 1a Inset; Supplementary Fig. 2b) but had limited neutralizing activity and failed to inhibit fusion (Fig. 1a, b). The neutralization of cell-free VZV and the fusion inhibition properties of mAb 93k and its Fab fragment confirmed that the $93 \mathrm{k}$ epitope was exposed in a prefusion conformation of $\mathrm{gB}$, indicating that $\mathrm{mAb} 93 \mathrm{k}$ could be used to identify residues involved in fusion initiation.

Near atomic resolution structure of the $\mathrm{gB}-93 \mathrm{k}$ interface. To define the molecular interactions formed between native $\mathrm{gB}$ and $\mathrm{mAb} 93 \mathrm{k}$, a $2.8-\AA$ structure of the gB-93k Fab complex was generated by using single particle cryo-EM, coupled with stringent particle selection and validation of local resolution $\left(\right.$ ResMap $\left.{ }^{34}\right)$, and feature resolvability through Q-score assignment (MapQ ${ }^{35}$ ) to amino acid side chains (Fig. 2a-d; Supplementary Figs. 1-3; Supplementary Tables 1-4; Supplementary Movie 1 and 2). Since gB was purified from VZV-infected MeWo cells, it was anticipated that alternative conformations of $\mathrm{gB}$ would be isolated, including prefusion forms. However, class averages were not identified in the cryo-EM micrographs that resembled a prefusion form of $\mathrm{gB}$ in complex with $\mathrm{mAb} 93 \mathrm{k}$ Fab fragments, underscoring the highly metastable status of the prefusion form of herpesvirus $\mathrm{gB}$. Local resolution of the cryo-EM map ranged from 2.3 to $4 \AA$ with expected visibility of the amino acid side-chain densities for the $\mathrm{gB}$ ectodomain to ensure the assignment of sequence identity (Fig. 2c, d; Supplementary Figs. $2 \mathrm{f}$ and 3). The VZV gB ectodomain was composed of five domains, DI to DV, with 13 helices and $31 \beta$-strands, similar to herpesvirus orthologue structures in their postfusion conformations (Fig. 2c, d; Supplementary Table 2). Each protomer was stabilized by five disulfide bonds, conserved among alpha-, beta-, and gammaherpesviruses (Fig. 2b, Supplementary Table 3). C122/ C584 stabilized the non-contiguous regions of DIV (N-terminal, aa115-136 and C-terminal, aa570-681) and C139/C540 bridged the distal end of the long central helix of DIII ( $\alpha 8)$ with the linker to DII. C213/C277, C369/C417, and C608/C645 formed stabilizing intra-domain interactions within domains I, II, and IV respectively.

The 2.8- $\AA$ cryo-EM map provided near atomic level details of the $\mathrm{mAb} 93 \mathrm{k}$ footprint on VZV gB identifying interactions between the variable heavy chain complementarity determining region 3 (VHCDR3) loop and regions in DIV, encompassing residues $589-597$ of $\beta 23,613-628$ of $\beta 25-\beta 26$, and $658-670$ of $\beta 28-30$ (Figs. 2d, 3, and 4; Supplementary Fig. 4; Supplementary Spreadsheet 1). On one side of VHCDR3, sidechains of I100, A102, A105, and Y113 formed a hydrophobic network with gB residues R592 and I594 of $\beta 23$, and V617 and L619 of $\beta 25$ (Figs. 3 and 4; Supplementary Movie 3; Supplementary Table 4). The sidechain of gB R592 formed a cation- $\pi$ interaction with the aromatic ring of VHCDR3 Y113 and fit into a negatively charged pocket within the 93k antigen binding site (Fig. 4b-d; Supplementary Movie 3 and 4). In addition, the carbonyl oxygen and backbone nitrogen of gB I593 and L595 formed H-bonding with the $\mathrm{OH}$ group of VHCDR3 Y113 and the sidechain of N111 respectively (Supplementary Movie 3 ). At the periphery of gB $\beta 23$ and 93k interface, the sidechain of the gB Q596 and the backbone nitrogen of N597 H-bonded with the carbonyl oxygen of 

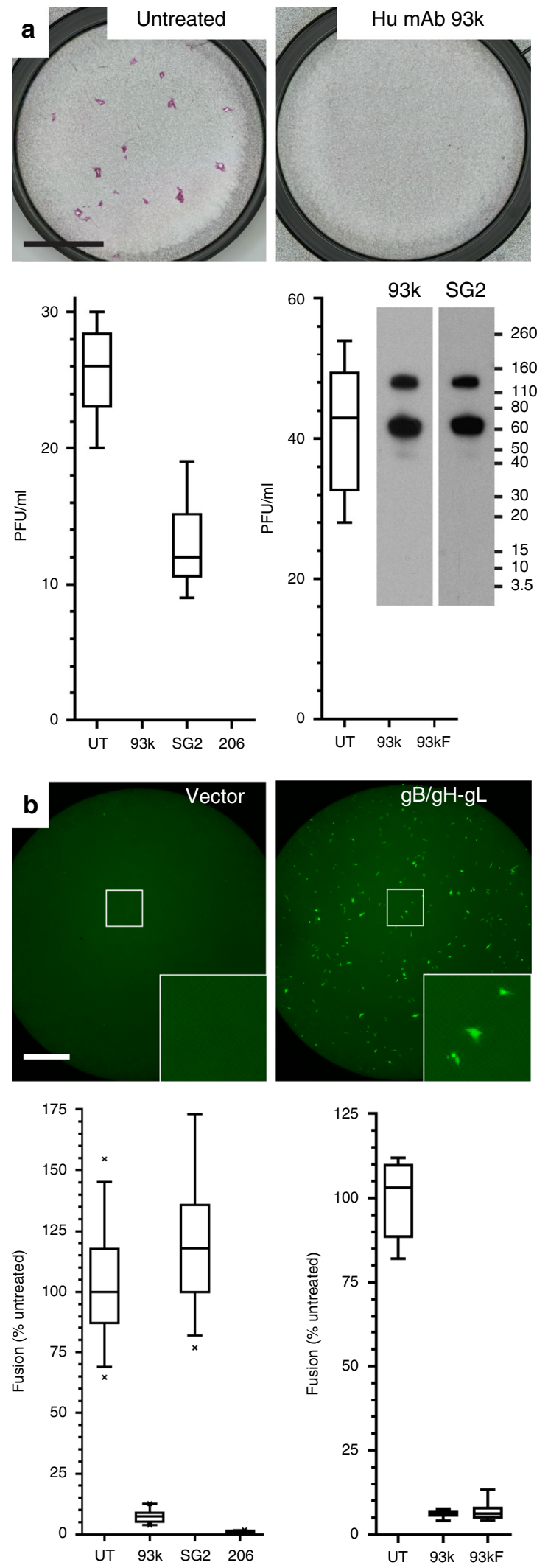

VHCDR3 residues P103 and G104, respectively, while the backbone nitrogen of VHCDR3 A106 H-bonded with the gB L595 carbonyl oxygen (Fig. 4c; Supplementary Movie 3). This marked a turning point of the interface after which residues of 93k interacted with $328-30$, dominated by hydrophobic and Van der Waals contacts. The H-bond between gB E670 OE1 and
Fig. 1 Human mAb 93k neutralizes VZV by fusion inhibition. a Cell-free VZV plaque neutralization assay. Immunohistochemical staining of VZVinfected MeWo cells untreated or treated with mAb 93k. Scale bar (black) $5 \mathrm{~mm}$. The box and whisker plots (5-95 percentile) of VZV neutralization with mAbs 93k, SG2 (murine anti-gB) and 206 (murine anti-gH), or 93k Fab fragments (93kF) represent $n=6$ samples examined over two independent experiments. Inset - western blots of purified, full-length VZV $\mathrm{gB}$ resolved by SDS-PAGE and detected using the mAbs 93k or SG2. Numbers represent molecular weights $(\mathrm{kDa})$ for the protein standard. VZV $\mathrm{gB}$ is cleaved by furin 33 ; both uncleaved $(130 \mathrm{kDa})$ and cleaved $(65 \mathrm{kDa}) \mathrm{gB}$ is detected by mAbs $93 \mathrm{k}$ and SG2. b Cell-cell fusion inhibition assay. Fluorescence microscopy of fused cells (GFP; green) generated by cotransfection of $\mathrm{gB} / \mathrm{gH}-\mathrm{gL}$ in the stable reporter fusion assay (SRFA). Scale bar (white) $1 \mathrm{~mm}$. The box and whisker plots (5-95\%) of fusion inhibition with mAbs 93k, SG2 and 206 or the 93kF represent $n=34$ (mAbs) or $n=$ 8 (Fab) samples examined over two independent experiments. Source data are provided as a Source Data file.

VHCDR3 T108 OG1 was surrounded by hydrophobic interactions formed by gB $328-30$ residues F655, H658, V660, and Y667, residues P107, P109, and L110 of VHCDR3, and W32 of the variable light chain CDR1 (VLCDR1; Fig. 4c; Supplementary Movie 3). Additional $\mathrm{H}$-bonds and salt bridges peripheral to the gB-93k $\beta$-sheet interactions were formed between sidechain atoms of gB T624 and VHCDR1 N31, gB R592 and VLCDR2 Y49, and gB S589 and VLCDR2 N56. Thus, the 2.8- $\AA$ cryo-EM map revealed a complex network of hydrophobic and hydrophilic interactions at the $\mathrm{gB}-93 \mathrm{k}$ interface of postfusion $\mathrm{gB}$ with the strongest interactions between $\mathrm{gB} \beta 23$ and $\beta 30$, and the $93 \mathrm{k}$ VHCDR3. Importantly, because $\mathrm{mAb} 93 \mathrm{k}$ has neutralizing activity through fusion inhibition (Fig. 1a, b), this suggested that residues within gB DIV $\beta 23$ and $\beta 30$ play a functional role in membrane fusion.

Domain IV $\beta 23$ residues are critical for $\mathrm{gB}$ fusion function. To determine their biological function, gB DIV $\beta 23$ residues were selected for alanine substitution, either individually or in combination and effects on $\mathrm{gB}$ binding to $\mathrm{mAb} 93 \mathrm{k}, \mathrm{gB} / \mathrm{gH}$-gLmediated membrane fusion and production of infectious VZV were evaluated. Residue S589 was used as a control because it was not a major contributor to the gB-93k interface. All of the single residue $\beta 23$ substitutions except S589A significantly reduced (R592A:17\%; I594A: 30\%, N597A:14\%) or abolished (Q596A) fusion compared to WT gB (100\%) but retained the capacity to traffic to cell surfaces and bind mAb 93k (Fig. 5a, b). The quantities of cell surface $\mathrm{gB}$ were lower for R592A (76\%), I594A (82\%), and Q596A (60\%) compared to WT (100\%) but gB levels were not diminished to amounts that might account for the markedly reduced fusion (Fig. 5b; Supplementary Fig. 5). Although mutations at the gB-93k interface might reduce the ability of $\mathrm{mAb} 93 \mathrm{k}$ to bind cell surface $\mathrm{gB}$, the difference in detectable levels of $\mathrm{gB}$ between $\mathrm{mAb}$ SG2 and mAb 93k could be related to prefusion and postfusion conformations of $\mathrm{gB}$. Mutants that reduce fusion could prevent the binding of $\mathrm{mAb}$ SG2 if its epitope was only exposed in a postfusion conformation, which is in contrast to mAb 93k because its epitope is accessible in both prefusion and postfusion conformations of $\mathrm{gB}$. Thus, in the context of the minimal $\mathrm{gB} / \mathrm{gH}$-gL fusion complex, the selected $\beta 23$ residues might play a significant role in membrane fusion.

To explore their role in virion entry fusion, the same $\beta 23$ mutations were transferred into the BAC-derived pOka-TK-GFP (designated WT) VZV (Supplementary Fig. 6A). Transfection with the WT BAC yields productive infection and spread by fusion of the virion envelope with adjacent uninfected cells, and 

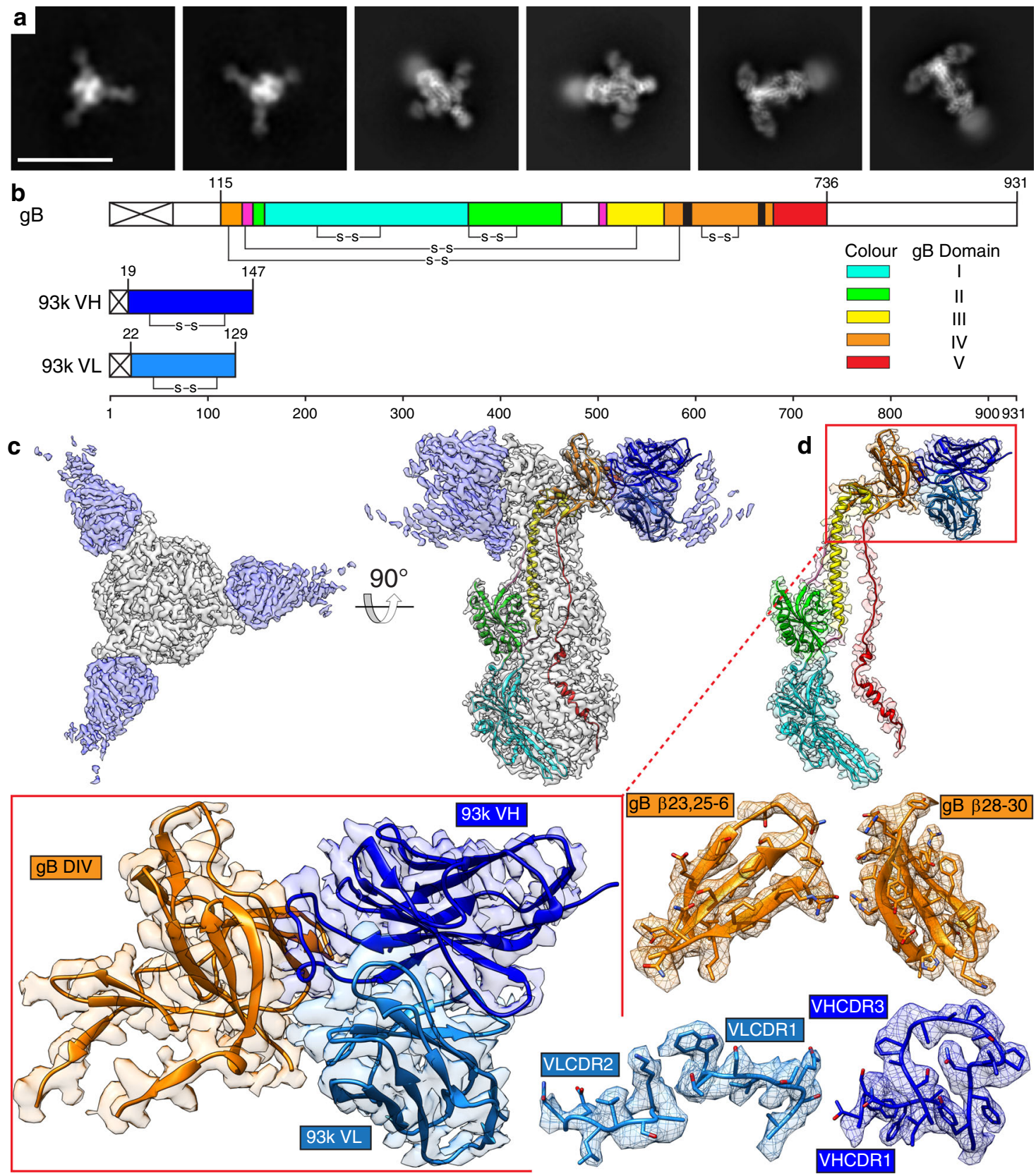

Fig. 2 Near atomic resolution cryo-EM structure of human neutralizing mAb 93k Fab fragments bound to native VZV gB. a Representative $2 D$ class averages used to generate the $2.8-\AA$ cryo-EM structure of the gB-93k complex. Scale bar (white) $20 \mathrm{~nm}$. $\mathbf{b}$ Linear maps of VZV gB, $93 \mathrm{k} V H$ and $93 \mathrm{k} V \mathrm{~L}$, are drawn to scale and each domain colored accordingly: DI (cyan), DII (green), DIII (yellow), DIV (orange), DV (red), linker regions (hot pink), 93k VH (blue) and 93k VL (light blue). The predominant interaction sites of the 93k VHCDR3 loop at gB $\beta 23$ and $\beta 30$ are highligted in black on the gB linear structure. c Cryo-EM map of the VZV gB-93k complex. The gB trimer (gray) and the 93k Fab fragments (blue) are segmented (see Supplementary Movie 1). d Segmentation of the cryo-EM map for one VH and VL chain of a 93k Fab fragment bound to a protomer of VZV gB (See Supplementary Movie 1). The structures of $\mathrm{VZV} g B$ and $93 \mathrm{k} V H$ and $V L$ are represented as ribbons. Expanded views of the cryo-EM map for gB DIV and the $93 \mathrm{k} V H$ and $V L$ are highlighted by the red boxes with the structure represented by ribbons and the cryo-EM map desity shown. Segementation and amino acid sidechains are shown for the exploded view of gB DIV $\beta$-strands ( $\beta 23,25-26$ and $\beta 28-30)$ and mAb 93k CDR loops (VHCDR1, VHCDR3, VLCDR1, and VLCDR2) that form the gB-93k interface (see Supplementary Movie 2).

polykaryocytes are formed due to cell fusion ${ }^{36}$. The gB $\beta 23$ mutant BACs with single residue substitutions, R592A, I594A, and Q596A, produced infectious VZV that spread in MeWo cell monolayers, as did the S589A control, indicating a less critical role for $\mathrm{gB}$-mediated virion entry and cell fusion in the presence of other VZV proteins than for fusion by the $\mathrm{gB} / \mathrm{gH}$-gL complex alone (Fig. 5b, c). Plaque sizes for Q596A but not the S589A, R592A, and I594A mutants were significantly smaller than WT
VZV (Fig. 5d, Supplementary Spreadsheet 2). However, mutations that significantly reduced fusion (R592A, I594A, and Q596A) all produced significantly smaller plaques compared to S589A when incorporated into the VZV genome. This differential reflected the findings of an increase (S589) or decreases (R592A, I594A, and Q596A) in fusion, suggesting that the $\mathrm{gB}$ point mutations had subtle effects on VZV replication. N597A was the only single residue mutation in $\beta 23$ that significantly reduced cell 
a
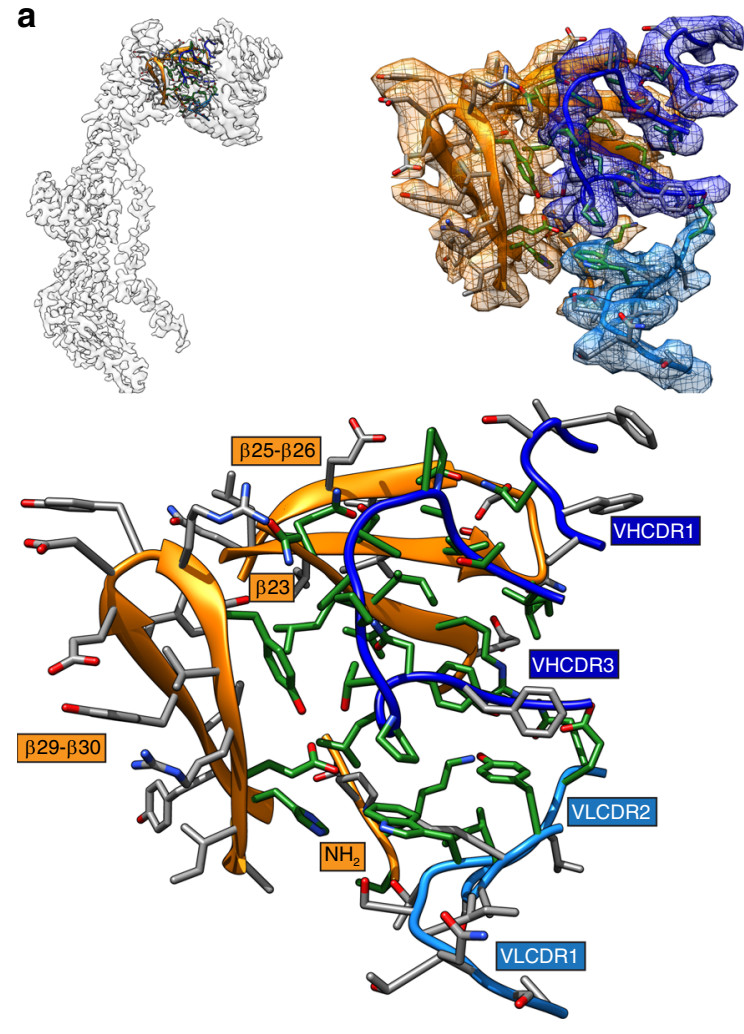

C

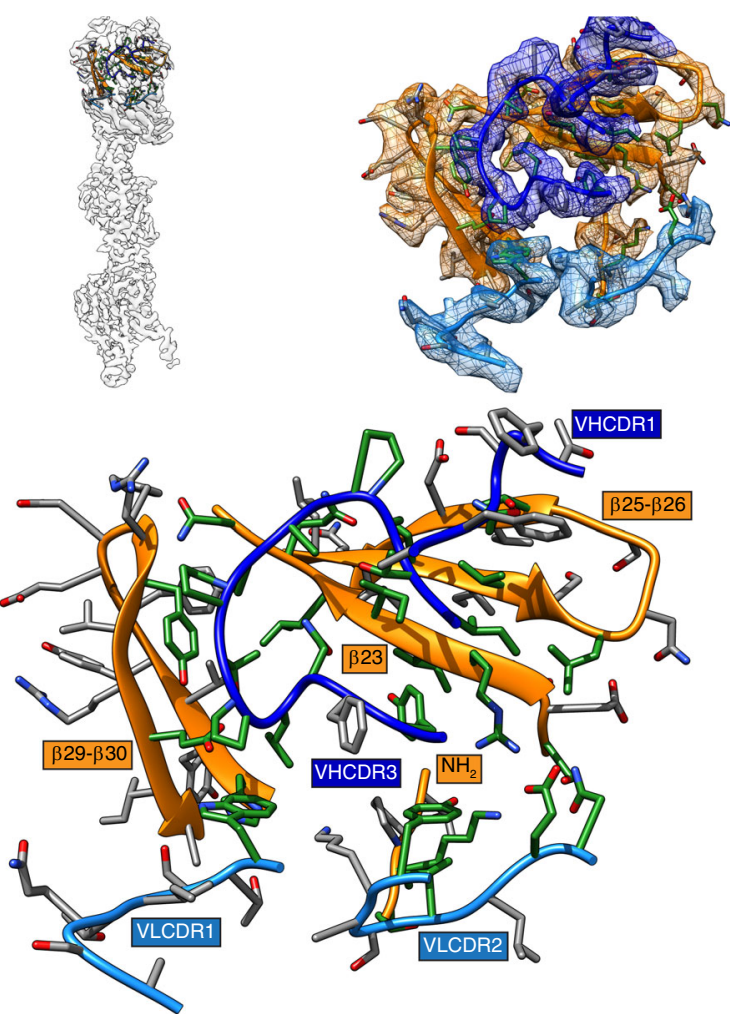

b
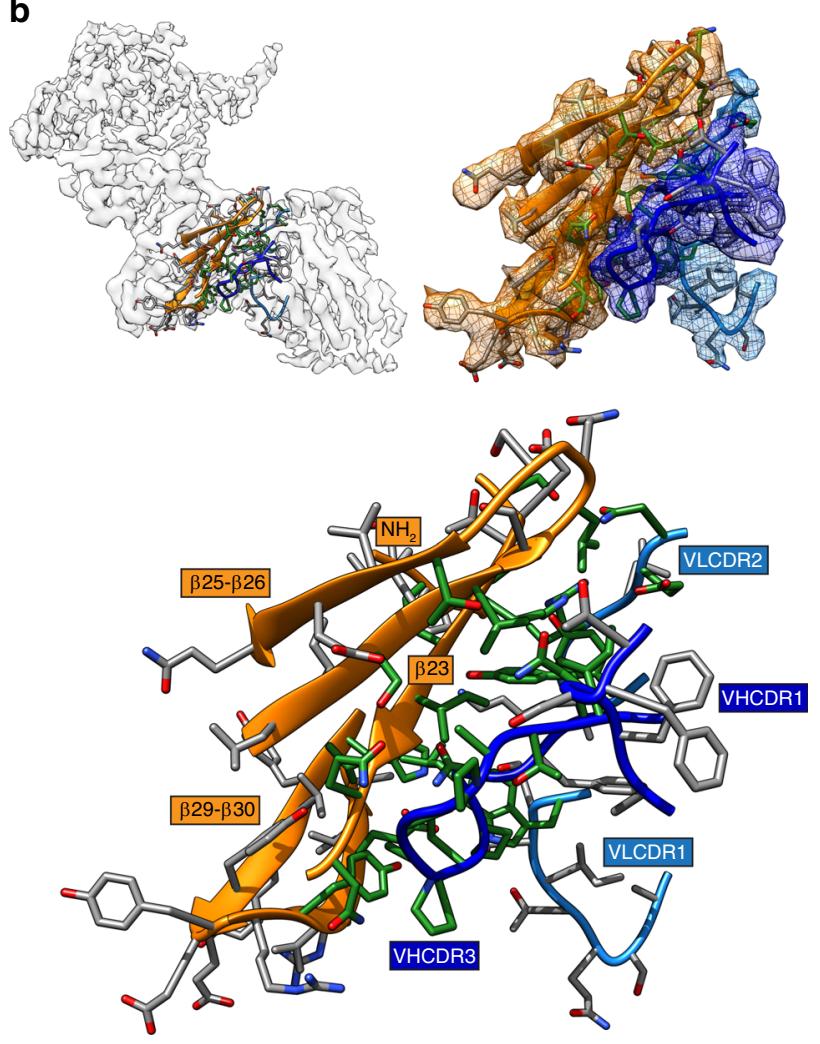

d
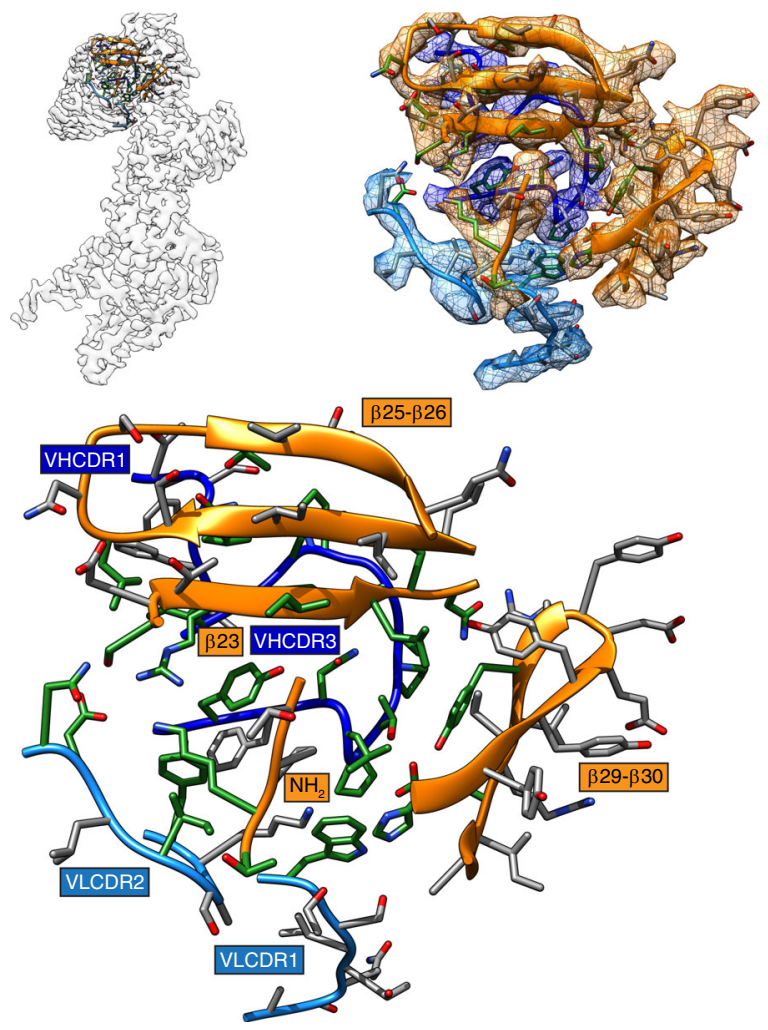

Fig. 3 Human mAb 93k forms a stable interface with gB Domain IV. a-d Location in the 2.8- $\AA$ cryo-EM map (top left panels) of the extracted densities (top right panels; scenes captured from Supplementary Movie 3) for the gB protomer and the associated bound 93k Fab. The densities of gB DIV (orange), $93 \mathrm{k}$ VH chain (blue) and $93 \mathrm{k} \mathrm{VL}$ chain (light blue) are highlighted. A ribbon diagram and side chains of the amino acids at the extracted densities are shown with those highlighted in green representing the interactions formed at the gB-93k interface. The bottom panels duplicate the regions in the top right panels but without the extracted cryo-EM map densities. The $\beta 23, \beta 25-26, \beta 29-30$, and the $\mathrm{NH}_{2}$ terminus of gB are highlighted with orange boxes, and the VHCDR1, VHCDR3, VLCDR1, and VLCDR2 are highlighted by blue boxes; VH - dark blue, VL - light blue. 
a

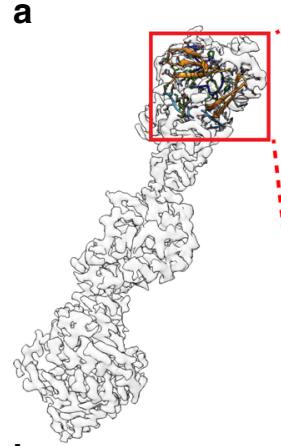

C

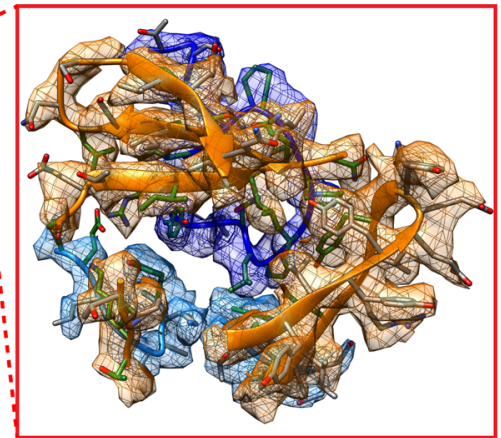

b

b $\beta 25-\beta 26$

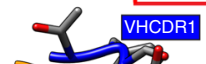

VHCDR1
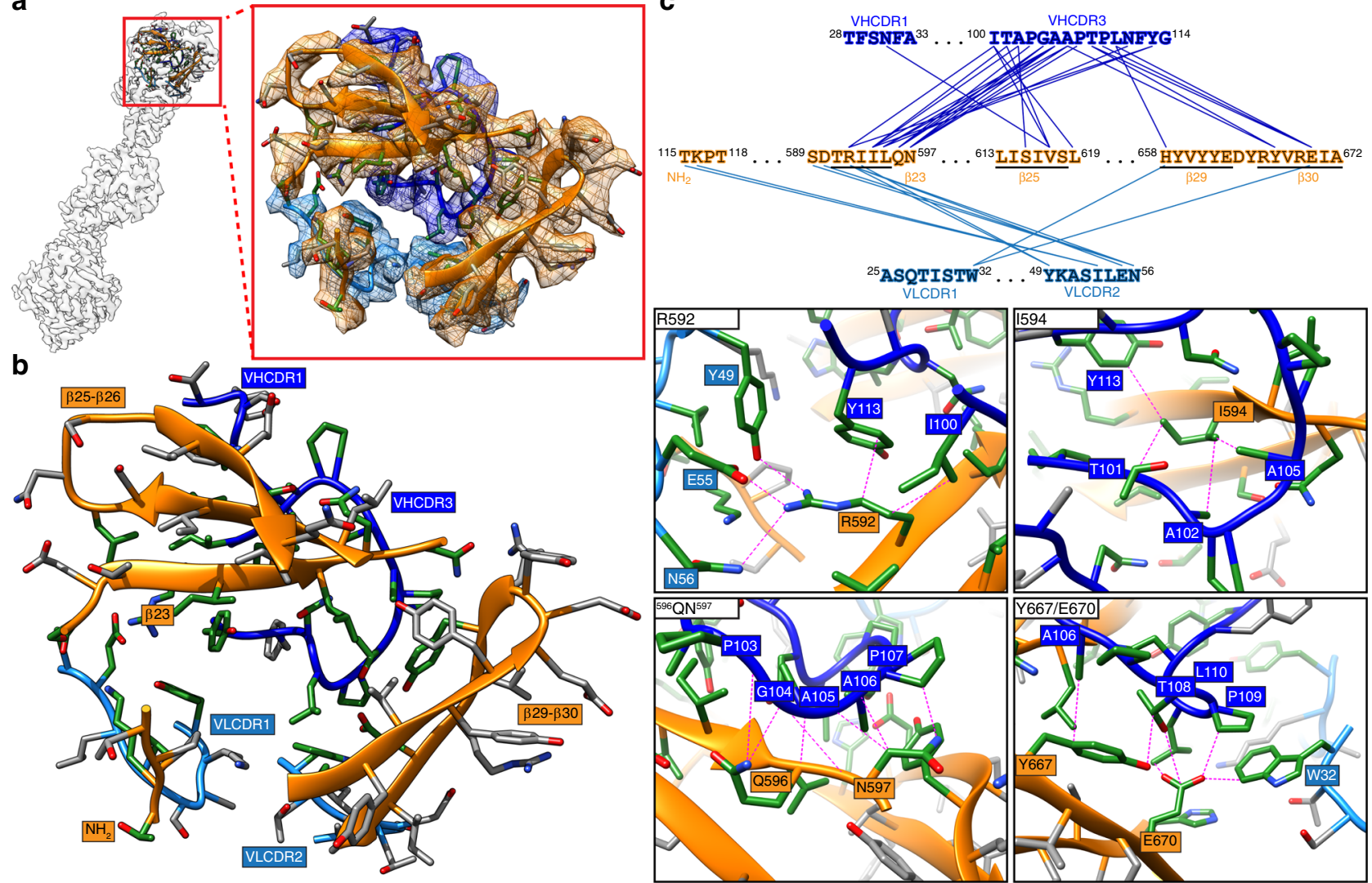

ASQTISTW ${ }^{32} \ldots{ }^{49}$ YKASIIEN $^{56}$

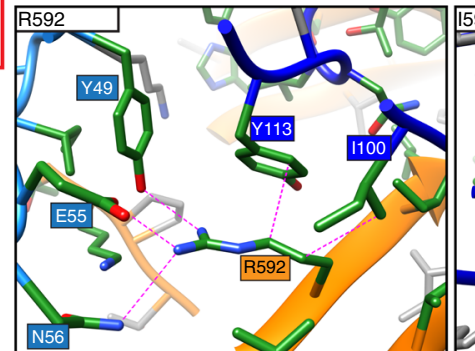

(1)

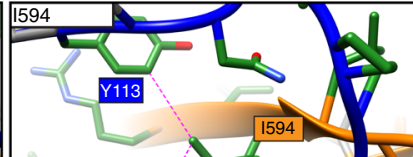

d

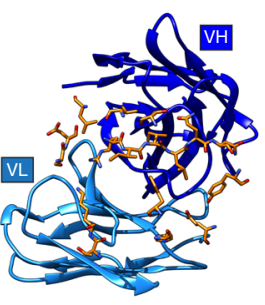

e
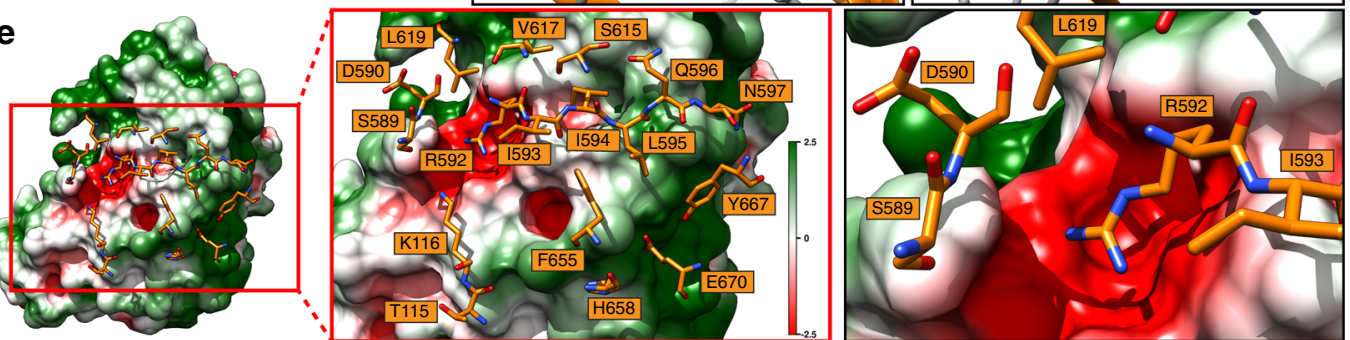

Fig. 4 Molecular interactions at the VZV gB-93k interface. a Location in the 2.8- $\AA$ cryo-EM map and extracted densities of one gB protomer in complex with a 93k Fab fragment. The zoomed in panel shows the extracted densities at the gB-93k interface. b, c Representative scenes from Supplementary Movie 3. b Ribbon diagram of the amino acids from the extracted density at the gB-93k interface (a). Those highlighted in green represent interacting amino acids. The $\beta 23, \beta 25-26, \beta 29-30$, and the $\mathrm{NH}_{2}$ terminus of $\mathrm{gB}$ are highlighted with orange boxes, and the VHCDR1, VHCDR3, VLCDR1, and VLCDR2 are highlighted by blue boxes; VH - dark blue, VL - light blue. c Molecular interactions between $\mathrm{gB}$ and the $\mathrm{VH}$ and $\mathrm{VL}$ chains of $\mathrm{mAb} 93 \mathrm{k}$. The upper panel shows a linear map of the interactions (blue lines) between $g B$ residues in $\beta 23, \beta 25, \beta 29$, and $\beta 30$ with those in VHCDR1, VHCDR3, VLCDR1, and VHCDR2 (not shown in Supplementary Movie 3). Underlined amino acids represent beta strands. The four lower panels show the interactions between gB residues R592, 1594, ${ }^{596} \mathrm{QN}^{597}$, and Y667/E670D with mAb 93k. Dotted lines (magenta) represent molecular interactions calculated using Find Contacts (Chimera, Supplementary Data 1). All interactions are shown in Supplementary Movie 3. d, e Orientations are equivalent to B. d The mAb 93k VH (blue) and VL (light blue) chains with gB interacting amino acid side chains (orange). e Surface electrostatic potential of the mAb $93 \mathrm{k}$ Fab VH and VL chains (calculated using APBS; Adaptive Poisson-Boltzmann Solver ${ }^{69}$ ) and zoomed in (middle and right panels) to highlight the $g B$ interface residues represented in stick format (orange), which are scenes from Supplementary Movie 4. The potentials are on a red-white-green color map (-2.5 to 2.5) in units of $\mathrm{kJ} / \mathrm{mol} / \mathrm{e}$.

fusion and prevented infection of neighboring cells. This effect was unrelated to glycosylation because the N597 was not part of an NXS/T N-linked glycosylation motif. Interestingly, this residue is preserved within the $\mathrm{gB}$ amino acid sequence NMSR in alphaherpesviruses, suggesting a conserved function.

In contrast to the single residue substitutions, combined substitutions in $\beta 23$, including ${ }^{589} \mathrm{AAA}^{594}$ (triple substitution: S589A/R592A/I594A), ${ }^{596} \mathrm{AA}^{597}$, and ${ }^{592} \mathrm{~A} /{ }^{596} \mathrm{AA}^{597}$, prevented both $\mathrm{gB}$-dependent fusion and productive infection (Fig. 5b, c). Of these, the ${ }^{589} \mathrm{AAA}^{594}$ mutant had less cell surface amounts of gB $(38 \%)$ and was the only mutant with significantly reduced quantities of total gB (66\%) compared to WT. However, other mutations with cell surface and total $\mathrm{gB}$ quantities in this range have been compatible with infection, suggesting that the fusion and infection deficiency was a direct effect of ${ }^{589} \mathrm{AAA}^{594}$ on $\mathrm{gB}$ function (Fig. 5c; Supplementary Fig. 5). Notably, transfection of the three $\beta 23$ mutant BACs that had combined substitutions produced single cells that expressed the VZV immediate early protein 62 (IE62) and TK-GFP (late protein). Spread to adjacent cells and syncytia formation was prevented by these substitutions, defining their importance for the fusogenic functions of $\mathrm{gB}$ during VZV infection. 
a
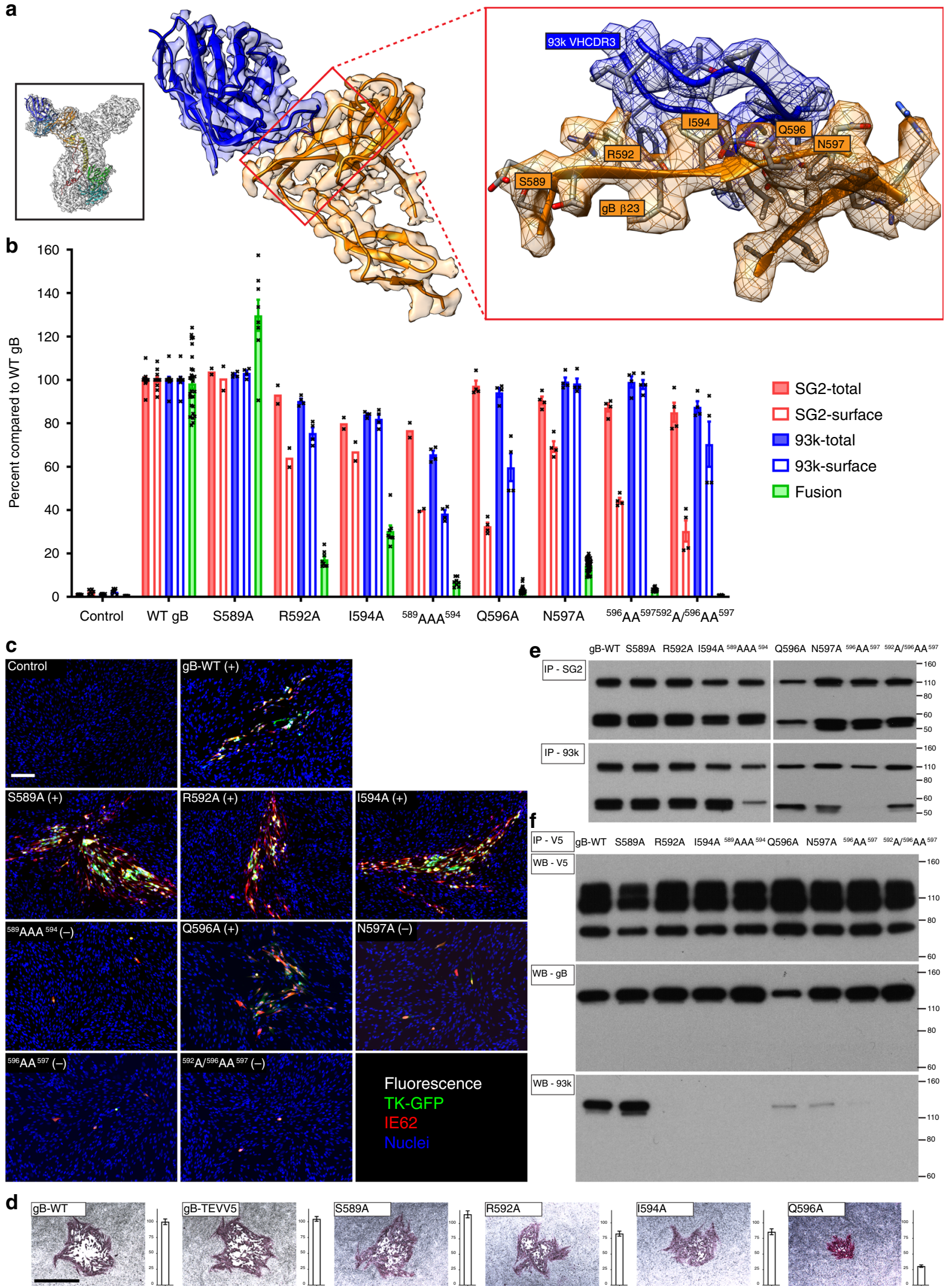

All of the gB $\beta 23$ mutants were immunoprecipitated with either mAbs 93k or SG2, under conditions to detect conformational epitopes (Fig. 5e). The quantity of the ${ }^{589} \mathrm{AAA}^{594}$ mutant was reduced for $93 \mathrm{k}$ compared with SG2, supporting the cryo-EM data that $\mathrm{gB} \beta 23$ contributes significantly to the gB-93k interface.
The $\mathrm{gB} / \mathrm{gH}$-gL fusion complex was immunoprecipitated using $\mathrm{mAb} 93 \mathrm{k}$ or an anti-V5 mAb to bind gH-V5 (Supplementary Fig. 7). As $m A b$ 93k binds to $g B$ within DIV, these data eliminated DIV as a region necessary for the interaction of $\mathrm{gB}$ with $\mathrm{gH}$-gL. Importantly, none of the $\mathrm{gB} \beta 23$ substitutions 
Fig. 5 Domain IV $\mathbf{\beta 2 3}$ residues are critical for fusion function and VZV replication. a Near atomic structure of the mAb $93 \mathrm{k}$ binding site at $V Z V \mathrm{gB} \beta 23$. The orientation of the complete gB-93k Fab map is shown in the small box in the top left-hand corner colored as for Fig. 1e. A portion of the cryo-EM map for gB domain IV chain A (Orange) and the bound 93k Fab (Blue) are shown. The red box on the right is a magnified view of $\beta 23$ with the amino acid side chains on VZV gB shown and those subjected to mutagenesis labeled. $\mathbf{b}$ Quantification of total and cell surface levels of gB DIV mutants produced by transfected $\mathrm{CHOs}$ and their capacity for cell-cell fusion measured by the SRFA. All values are normalized as a percentage to WT gB. Bar charts represent $n=4$ ( $n=2$ for SG2 staining of S589A, R592A, 1594A, and ${ }^{589}$ AAA $^{594}$ ) samples for total and cell surface gB detected using mAbs SG2 and $93 \mathrm{k}$, and $n=$ $28\left(n=8\right.$ for S589A, R592A, 1594A, and ${ }^{589} \mathrm{AAA}^{594}$ ) samples for fusion examined over two independent experiments. Error bars represent \pm SEM. c Immunofluorescence of MeWo cells at $72 \mathrm{~h}$ post transfection with pOka-BACs with gB mutations. Melanoma cells were transfected with pOka-TK-GFP BACs carrying alanine substitution S589A, R592A, 1594A, ${ }^{589}$ AAA 594, Q596A, N597A, ${ }^{596}$ AA 597 , and ${ }^{592} \mathrm{~A} /{ }^{596} \mathrm{AA}{ }^{597}$. The (+) or (-) indicate whether or not virus was recovered from the transfections. Immunofluorescence staining was performed for IE62 as a marker for early infection because the TK-GFP is a late protein product during VZV replication. Scale bar (white) $100 \mu \mathrm{m}$. d Immunohistochemistry staining of plaques and their sizes for the pOka-TK-GFP gB, pOka-TK-GFP gB-TEVV5, and $\beta 23$ mutants S589A, R592A, 1594A, and Q596A. Scale bar (black) $1 \mathrm{~mm}$. Bar charts represent $n=40$ plaques measured over two independent experiments (Supplementary Data 2). All values were normalized to WT VZV. Error bars represent \pm SEM. e Immunoprecipitation of the VZV gB $\beta 23$ mutants from transfected $\mathrm{CHO}$ cells using anti gB mAbs SG2 and 93k, and western blot with anti-gB Ab 746-868. The $\mathrm{gH}$ control lane where $\mathrm{CHO}$ cells were transfected with gH-WT is shown in Fig. 6e. f Reducing SDS-PAGE and western blot of gB coimmunoprecipitated with $\mathrm{gH}-\mathrm{V} 5$ from $\mathrm{CHO}$ cells transfected with the $\beta 23$ mutants, gH-V5 and gL. The gB-WT control lane using the gH-WT negative control is shown in Fig. 6f. Western blots were performed using mAb to V5 (Top), anti-gB Ab 746-868 (middle), and a mAb 93k (bottom). e, $\mathbf{f}$ Numbers to the right of the blots are molecular weight standards $(\mathrm{kDa})$. Source data are provided as a Source Data file.

prevented immunoprecipitation of the $\mathrm{gB} / \mathrm{gH}-\mathrm{gL}$ complex (Fig. 5f). In contrast to immunoprecipitation, $\mathrm{mAb} 93 \mathrm{k}$ did not bind to the gB R592A, I594A, or ${ }^{589}{ }^{A A A}{ }^{594}$ mutants in western blots and was reduced but not abolished for the Q596A, N597A, or ${ }^{596} \mathrm{AA}^{597}$ mutants, suggesting that R592 and I594 contributed more substantially to the $\mathrm{gB}-93 \mathrm{k}$ interaction.

Domain IV $\boldsymbol{\beta} 30$ residues are critical for fusion function. Based on the cryo-EM structure, interface residues Y667 and E670 located within gB DIV $\beta 30$ were also evaluated for their contribution to $\mathrm{gB}$ fusion function (Fig. $6 \mathrm{a}, \mathrm{b}$ ). Single substitutions, Y667A and E670A, reduced fusion $(<20 \%$ of $\mathrm{WT} g B)$ and a double substitution, ${ }^{667} \mathrm{~A} / \mathrm{A}^{670}$, abolished fusion. The limited cell surface quantity of the E670A mutant (30\% of WT gB) might contribute to its reduced fusion capacity (Fig. 6b; Supplementary Fig. 8). Y667A and ${ }^{667} \mathrm{~A} / \mathrm{A}^{670}$ mutant BACs both yielded infectious virus that spread to adjacent cells whereas the E670A mutant did not (Fig. 6c; Supplementary Fig. 6B). The plaque sizes for the $\mathrm{Y} 667 \mathrm{~A}$ and ${ }^{667} \mathrm{~A} / \mathrm{A}^{670} \mathrm{VZV}$ mutants were similar to both WT and S589A (Fig. 6d). However, when substitutions of the $\beta 23$ and $\beta 30$ interface residues were combined in the ${ }^{592} \mathrm{~A} /{ }^{596} \mathrm{AA}^{597} /{ }^{667} \mathrm{~A} / \mathrm{A}^{670}$ mutant, gB expression was not affected but $\mathrm{gB} / \mathrm{gH}-\mathrm{gL}$ fusion was undetectable and VZV spread was prevented (Fig. $6 \mathrm{~b}, \mathrm{c}, \mathrm{f}$ ).

The single Y667A and E670A mutations and the dual, ${ }^{667} \mathrm{~A} /$ $\mathrm{A}^{670}$, substitutions reduced but did not abolish mAb 93k binding as assessed by immunoprecipitation of gB (Fig. 6e). However, $\mathrm{mAb} 93 \mathrm{k}$ did not bind to the Y667A, E670A, and ${ }^{667} \mathrm{~A} / \mathrm{A}^{670}$ mutants in western blots, corroborating their role at the gB-93k interface. The combined $\beta 23 / \beta 30$ mutant, ${ }^{592} \mathrm{~A} /{ }^{596} \mathrm{AA} \mathrm{S}^{59} / 667 \mathrm{~A} /$ $A^{670}$, was not immunoprecipitated with $m A b 93 \mathrm{k}$, verifying the significance of these $\mathrm{gB}$ residues for $\mathrm{mAb} 93 \mathrm{k}$ binding (Fig. 6e, f). In addition, the failure of SG2 to immunoprecipitate this mutant confirmed that gB DIV residues contribute to the SG2 epitope. Similar to $\beta 23$, the $\beta 30$ substitutions alone did not prevent the coimmunoprecipitation of $\mathrm{gB}$ with $\mathrm{gH}-\mathrm{gL}$, showing that this region of $\mathrm{gB}$ DIV was not essential for the formation of the fusion complex.

The conserved conformation of VZV $\beta 30$ in the structures of the $\mathrm{gB}$ orthologues from HSV-1, PRV, HCMV, and EBV $2,4,5,37$ implies a pan-herpesvirus role of this structural element in $\mathrm{gB}$ mediated fusion, demonstrated by mAb 93k binding and at the functional level by the VZV mutagenesis data (Figs. 7 and 8). In contrast, the less conserved nature of the region corresponding to
VZV $\beta 23$ in the $\mathrm{gB}$ homologs suggests that it has evolved independently and has a virus-specific function in fusion which has diverged across the Herpesviridae.

\section{Discussion}

Membrane fusion for all herpesviruses is thought to be initiated by interactions between $\mathrm{gB}$ and $\mathrm{gH}-\mathrm{gL}$ on the virion surface to prime the $\mathrm{gB}$ trimer for fusion function ${ }^{9}$. When the fusion reaction is triggered, the fusion loops of $\mathrm{gB} \mathrm{DI}$ are proposed to embed into the opposing membrane via an as yet undefined molecular process that enables gB to bridge the two membranes and bring them together in a condensation reaction. In the present study, mutagenesis based on the 2.8 - $\AA$ cryo-EM structure of $\mathrm{gB}$ in complex with the potent neutralizing mAb 93k illuminates $\mathrm{gB}$ DIV as having a central role in the fusion reaction. This finding is broadly relevant as the $\mathrm{gB}$ fusogen is highly conserved and required for virion entry by all herpesviruses.

Since gB orthologues are classified as class III fusogens, current models of herpesvirus membrane fusion have been based on Xray crystallography data for the prototype class III fusogen VSV $\mathrm{G}$, including models of how $\mathrm{gB}$ orthologues transition from a putative prefusion to a postfusion conformation ${ }^{6,7,9}$. However, there are significant differences between these trimeric viral fusogens. Importantly, the biochemical requirements of herpesvirus $\mathrm{gB}$ orthologues are more complex than VSV G because interactions with the $\mathrm{gH}$-gL heterodimer are necessary to prime $\mathrm{gB}$ for fusion ${ }^{38,39}$. Moreover, the quaternary structures of herpesvirus gB and VSV G DIV differ ${ }^{2-6,10,11}$, consistent with likely variations in the molecular mechanisms that mediate the fusion reaction. It has been challenging to determine the role of herpesvirus gB DIV in membrane fusion owing to the low-resolution cryo-EM structures for DIV in proposed prefusion conformations and its reported intrinsic flexibility ${ }^{10,40-42}$

Based on the findings of the present study, we propose that the quaternary structure encompassing gB DIV, referred to as the crown $^{5}$, remains intact during the transition of $\mathrm{gB}$ from prefusion conformations to its postfusion state. This is substantiated by key structural and biochemical observations. Firstly, the cryo-EM structure of the gB-93k Fab interface shows that the conformation of gB DIV was similar to that of the postfusion state $e^{2-5}$, while the fusion inhibition properties of $\mathrm{mAb} 93 \mathrm{k}$ authenticates the accessibility of DIV in a prefusion form of $\mathrm{gB}$ (Figs. 1-4). Importantly, 93k can bind to $\mathrm{gB}$ when it is in complex with the fusion priming heterodimer gH-gL (Supplementary Fig. 7). This implies that both $\mathrm{gB} \beta 23$ and $\beta 30$, central to the 93k epitope, 
a

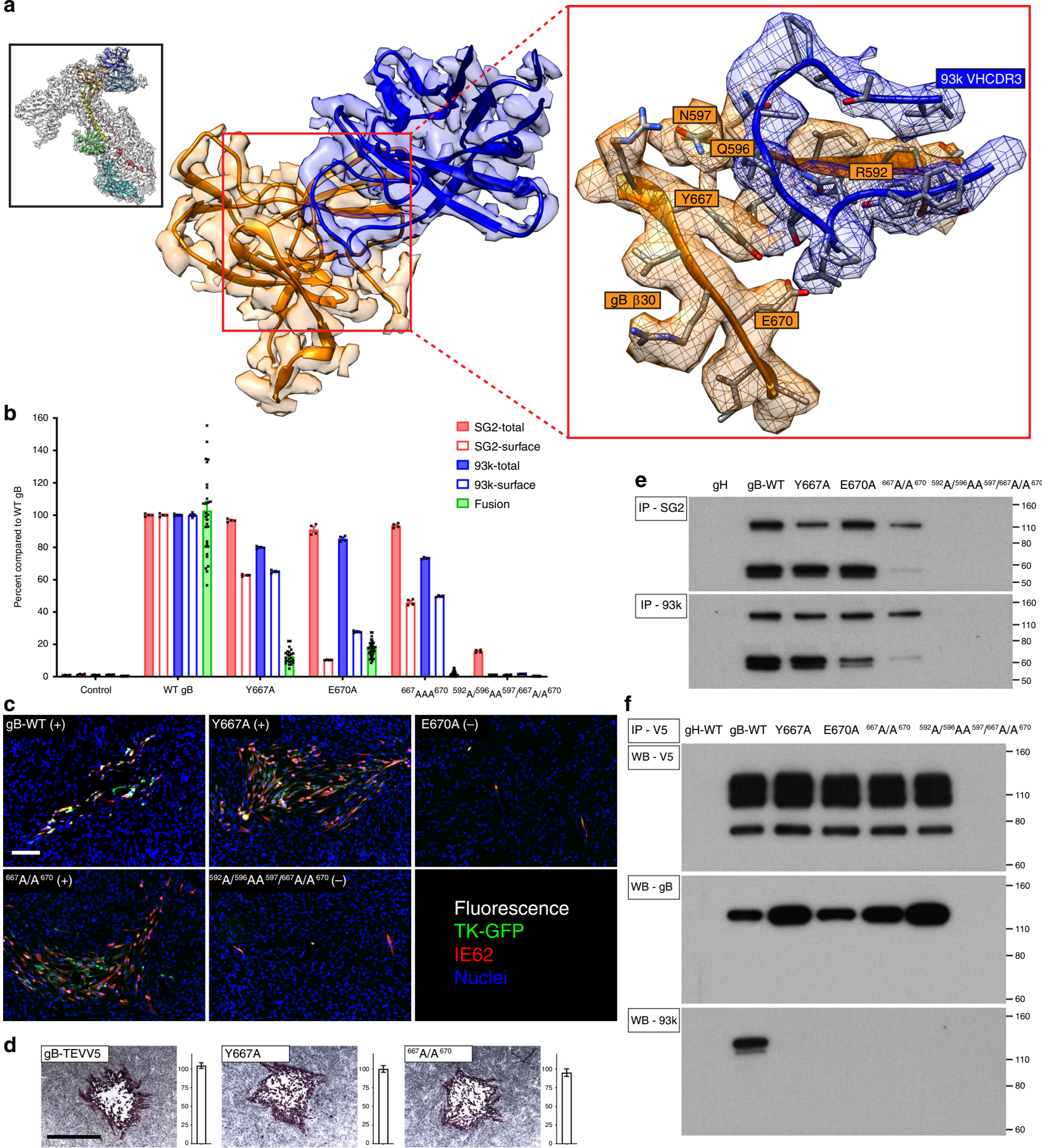

maintain their conformations in pre- and postfusion states of $\mathrm{gB}$. As noted, the beta strand equivalent to VZV $\beta 30$ remains structurally conserved in the herpesvirus gB orthologues, suggesting a conserved function in fusion (Fig. 8). Secondly, two cysteine bonds, conserved in the Herpesviridae ${ }^{2-5}$, are formed within DIV of each gB protomer at ${ }^{122} \mathrm{C}-\mathrm{C}^{584}$ and ${ }^{608} \mathrm{C}-\mathrm{C}^{645}$. The ${ }^{122} \mathrm{C}-\mathrm{C}^{584}$ bond stabilizes the $\mathrm{N}$-terminal (aa115-136) and C-terminal (aa570-681) regions of $\mathrm{gB}$ DIV. An additional cysteine bond $\left({ }^{139} \mathrm{C}-\mathrm{C}^{540}\right)$ forms a stabilizing link between the DII N-terminal linker of $\mathrm{gB}$ and the distal region of DIII adjacent to DIV. These cysteine bonds ensure that gB DIV forms a tertiary structure similar to the conformation of VSV G DIV, which is maintained in both the pre- and postfusion structures ${ }^{6,7}$. Finally, the quaternary structure of the $\mathrm{gB}$ crown, which is comprised of DIVs from three protomers, differs structurally from VSV G. The gB crown is elevated above DIII and each DIV from a protomer in the gB trimer co-assembles to form the crown. This contrasts with VSV G where the DIV protomers remain as individual tertiary structures adjacent to DIII to form the crown ${ }^{6,7}$. Within each protomer, VSV G DIV also remains in close proximity to DII in the pre- and postfusion structures whereas the herpesvirus $\mathrm{gB}$ DIV is located away from and opposite to DII within the gB 
Fig. $\mathbf{6}$ Domain IV $\boldsymbol{\beta} \mathbf{3 0}$ residues are critical for fusion function. a Near atomic structure of the mAb 93k binding site at VZV gB $\beta 30$. The orientation of the complete gB-93k map is shown in the small box in the top left-hand corner colored as for Fig. 1e. A portion of the cryo-EM map for gB domain IV chain $A$ (orange) and the bound 93k Fab (blue) are shown. The red box on the right is a magnified view of $\beta 30$ with the amino acid side chains on VZV gB shown and those subject to mutagenesis labeled. b Quantification of total and cell surface levels of gB DIV mutants produced by transfected CHOs and their capacity for cell-cell fusion measured by the SRFA. All values are normalized as a percentage to WT gB. Bar charts represent $n=4$ samples for total and cell surface gB detected using mAbs SG2 and 93k, and $n=24$ ( $n=36$ for E670A) samples for fusion examined over two independent experiments. Error bars represent \pm SEM. $\mathbf{c}$ Immunofluorescence of MeWo cells at $72 \mathrm{~h}$ post transfection with pOka-BACs with gB mutations. Melanoma cells were transfected with pOka-TK-GFP BACs carrying alanine substitutions Y667A, E670A, ${ }^{667} \mathrm{~A} / \mathrm{A} / 670$, and ${ }^{592} \mathrm{~A} /{ }^{596} \mathrm{AA} \mathrm{A}^{597} /{ }^{667} \mathrm{~A} / \mathrm{A}^{670}$. The $(+)$ or $(-)$ indicate whether virus was recovered or not, respectively, from the transfections. Immunofluorescence staining was performed for IE62 as a marker for early infection because the TK-GFP is a late protein product during VZV replication. Scale bar (white) $100 \mu \mathrm{m}$. d Immunohistochemistry staining of plaques and their sizes for the pOka-TK-GFP gB, pOka-TK-GFP gB-STEVV5, and $\beta 30$ mutants Y667A and ${ }^{667} \mathrm{~A} / \mathrm{A}^{670}$. Scale bar (black) $1 \mathrm{~mm}$. Bar charts represent $n=40$ plaques measured over two independent experiments. All values were normalized to WT VZV (see Fig. $5 \mathrm{~d}$ ). Error bars represent \pm SEM.

e Immunoprecipitation of the VZV gB $\beta 23$ mutants from transfected CHO cells using anti gB mAbs SG2 and 93k, and western blot with anti-gB Ab 746-868. The $\mathrm{gH}$ lane is a control where $\mathrm{CHO}$ cells were transfected with gH-WT. $\mathbf{f}$ Reducing SDS-PAGE and western blot of gB co-immunoprecipitated with $\mathrm{gH}-\mathrm{V} 5$ from $\mathrm{CHO}$ cells transfected with the $\beta 23$ mutants, gH-V5 and gL. The first gB-WT lane is a control lane using gH-WT. Western blots were performed using mAb to V5 (Top), anti-gB Ab 746-868 (middle), and a mAb 93k (bottom). e, $\mathbf{f}$ Numbers to the right of the blots are molecular weight standards $(\mathrm{kDa})$. Source data are provided as a Source Data file.
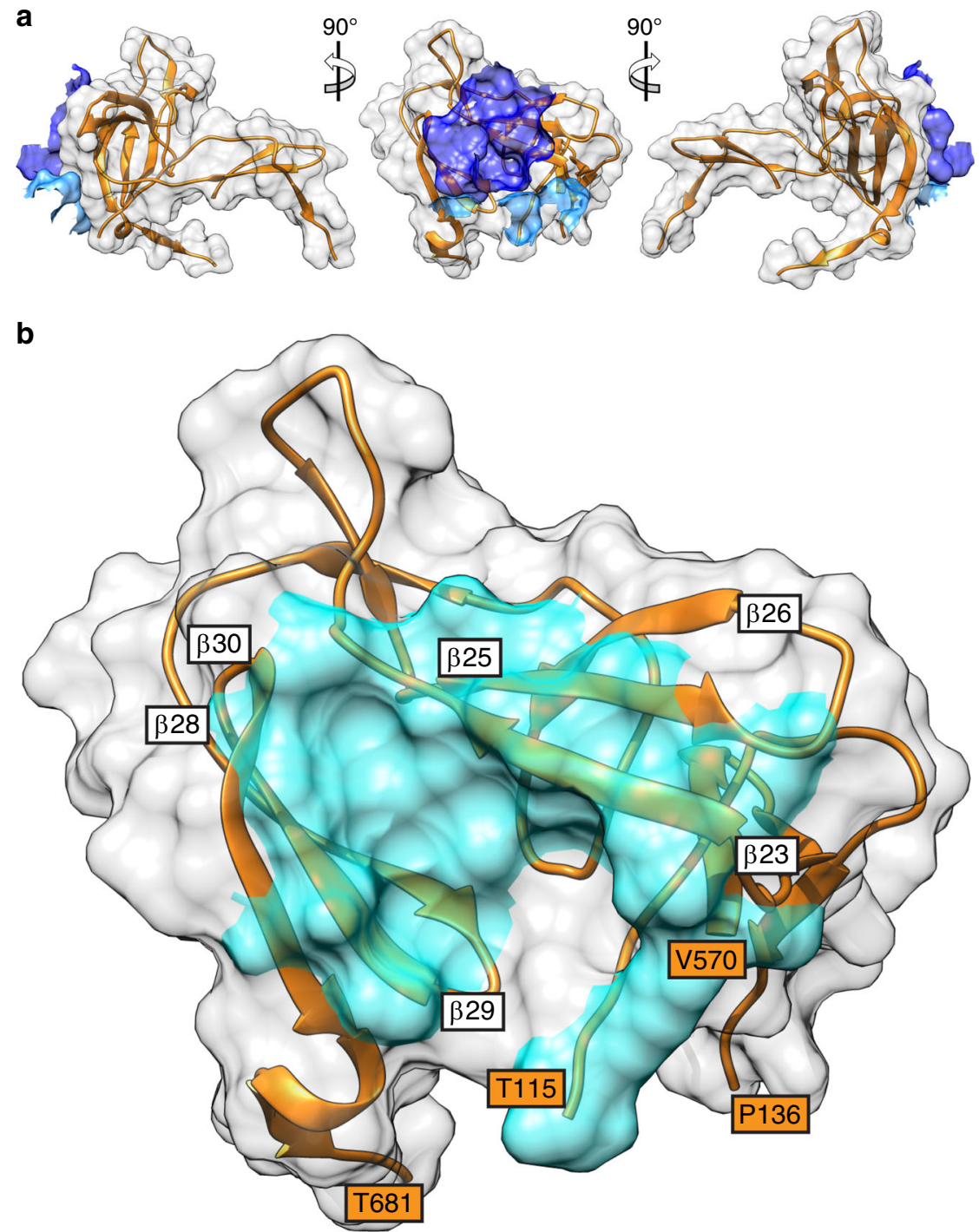

Fig. 7 The footprint of human mAb 93k on VZV gB DIV. a Surface rendering (gray) of VZV gB DIV (orange ribbon diagram) and the mAb 93k residues (dark blue - VH chain; light blue - VL chain) that form molecular contacts at the gB-93k interface. $\mathbf{b}$ Surface rendering of the mAb $93 \mathrm{k}$ footprint (cyan) on gB DIV (gray surface; orange ribbon diagram). The amino acids that demarcate the boundaries of $g B$ DIV are shown in orange boxes. The beta strands $\beta 23$, 25-26 and $\beta 28-30$ that were used to align $\mathrm{VZV} \mathrm{gB}$ with herpesvirus homologs (Fig. 8) are shown in white boxes at the $\mathrm{NH}_{2}$ terminus of the corresponding beta strand. 

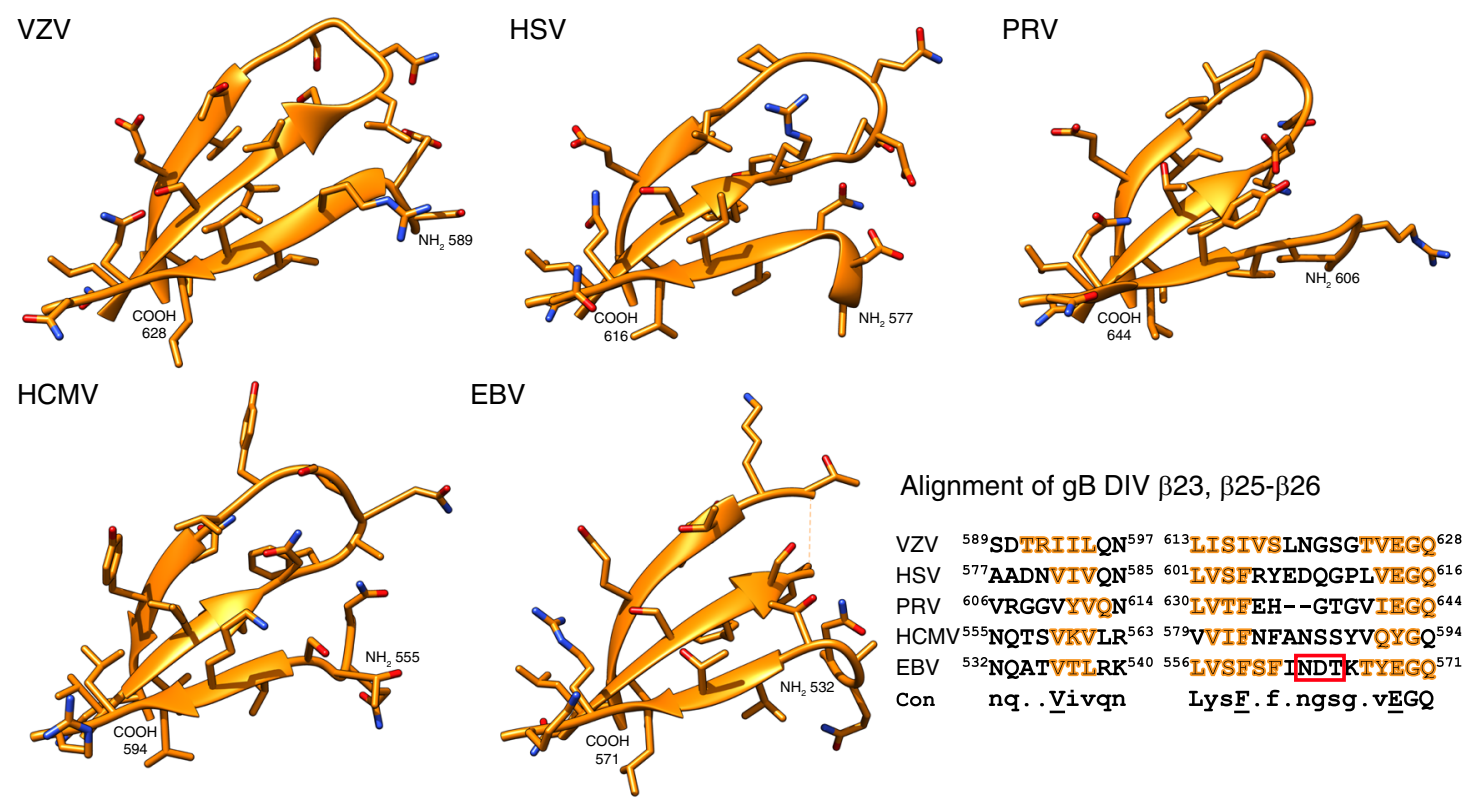

Alignment of gB DIV $\beta 23, \beta 25-\beta 26$

VZV ${ }^{589}$ SDTRIILQN ${ }^{597}{ }^{613}$ LISIVSLNGSGTVEGQ ${ }^{628}$ HSV ${ }^{577}$ AADNVIVQN ${ }^{585}{ }^{601}$ LVSERYEDQGPLVEGQ ${ }^{616}$ PRV ${ }^{606}$ VRGGVYVQN ${ }^{614}{ }^{630}$ LVTEEH--GTGVIEGQ ${ }^{644}$ HCMV ${ }^{555}{ }^{5} Q T S V K V L R^{563}{ }^{579}$ VVIFNFANSSYVQYGQ $Q^{594}$ EBV ${ }^{532}$ NQATVTLRK ${ }^{540}{ }^{556}$ LVSFSF INDTKTYEGQ ${ }^{571}$ Con nq. . Vivqn Lys $\underline{F} . f . n g s g . v E G Q$

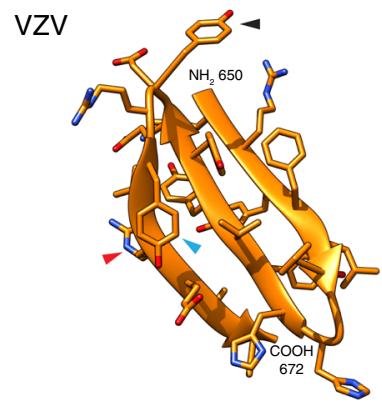

HSV
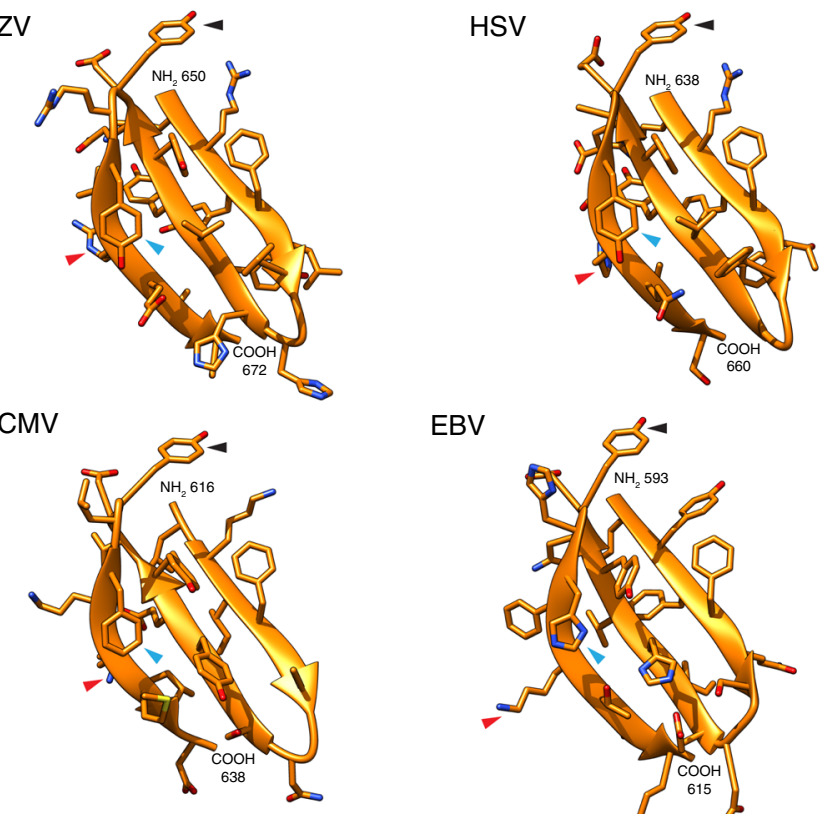

EBV

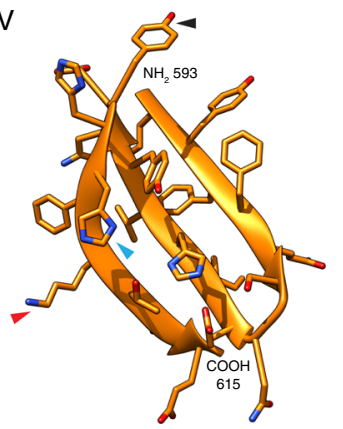

PRV

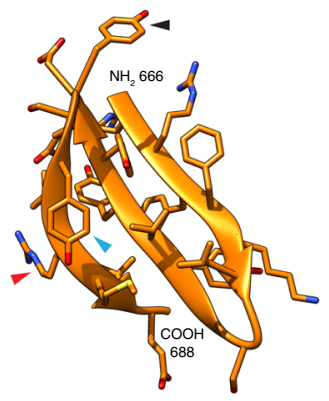

Alignment of gB DIV $\beta 28-\beta 30$

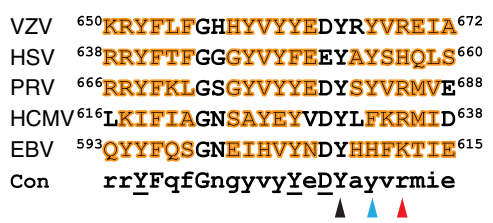

VZV ${ }^{650}$ KRYFLFGHHYVYYEDYRYVREIA ${ }^{672}$ HSV ${ }^{638}$ RRYETEGGGYVYFEEYAYSHQLS ${ }^{660}$ PRV ${ }^{666}{ }^{6 R Y F K L G S G Y V Y Y E D Y S Y V R M V E ~}{ }^{688}$ HCMV ${ }^{616}$ LKIFIAGNSAYEYVDYLFKRMID ${ }^{638}$

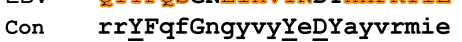
$\triangle \Lambda \Lambda$

Fig. 8 The VZV gB DIV $\boldsymbol{\beta} 30$ fold is conserved across the Herpesviridae. Structure alignments of VZV gB DIV encompassing $\beta$-strands 23 and $25-26$, and 28-30 with those of HSV (PDB 2GUM), PRV (PDB 6ESC), HCMV (PDB 5C6T), and EBV (PDB 3FVC). The $\beta$ strands are represented in ribbon format with the side chains shown. The amino acid alignments are shown in the lower right panel with each $\beta$-strand highlighted in orange. The consensus sequence (Con) shows conserved residues in upper case with those underlined showing conservations with the exception of one sequence. The red box for the EBV $\beta 23$ and 25-26 alignment highlights the region not resolved in the crystal structure of 3FVC. Arrow heads highlight the conserved tyrosine (black), conserved ring structure (blue), and conserved charge (red) in $\beta 30$.

protomer, further supporting the notion that the crown of $\mathrm{gB}$ orthologues remains as a single quaternary structure during the prefusion to postfusion transition.

Functional data derived from the virus-free fusion assay and VZV BAC transfection demonstrated that most DIV point mutations were tolerated in the context of VZV infection. This is consistent with previous work investigating the effects of $\mathrm{gB}$ point mutations on herpesvirus fusion function ${ }^{37}$. The exceptions in the present study were N597 and E670, which, considering the gB structure, might have roles in currently unexplored protein-protein interactions. In contrast, combined substitutions of two or more of the $\beta 23$ and $\beta 30$ residues reduced or abolished fusion and limited the capacity of VZV to infect cells, indicating that these residues act together to ensure that the $\mathrm{gB}$ structure supports its fusion function. The greater tolerance for DIV mutations in the context of VZV infection suggests that other viral proteins might act as accessory molecules to facilitate gBdependent fusion as reported for other herpesviruses, such as HSV-1 gD, HCMV gO or UL128-131, and EBV gp42 $43-45$. VZV gB might also interact with cell surface proteins as has been demonstrated for HSV-1 (PILRa), HCMV (PDGFRa), and EBV (NRP1; neuropilin 1) ${ }^{46-49}$, although gB domains important for these interactions have not been identified by mutagenesis or other methods. While VZV gB binding to myelin associated 
glycoprotein (MAG) has been described ${ }^{30}$, the interaction was not specific as VZV gE also bound to MAG ${ }^{50}$. Notably, coimmunoprecipitation of the $\beta 23$ and $\beta 30$ mutants demonstrated that this region of DIV was not required for $\mathrm{gB}$ binding to $\mathrm{gH}$-gL, providing further evidence that this subdomain is present on the prefusion form of $\mathrm{gB}$ and the interaction with $\mathrm{gH}$-gL does not mask DIV during fusion. This finding is consistent with a lowresolution structure indicating that $\mathrm{gH}$-gL binds to a putative prefusion form of $\mathrm{gB}$ on the surface of herpesvirus particles ${ }^{13}$.

Neutralizing antibodies also target the HSV-1 and HCMV gB DIV, indicating that the accessible residues of prefusion forms of $\mathrm{gB}$ are of broad importance for herpesvirus fusion function ${ }^{4,14-19}$. HSV-1 was neutralized by murine mAbs, SS10 and SS63, produced in mice vaccinated with $\mathrm{gB}^{15}$. Although the location of the SS63 epitope could not be defined further than DIV, truncation mutants of $\mathrm{gB}$ and low-resolution mapping by negative stain $\mathrm{EM}$ approximated SS10 binding to HSV-1 gB residues $640-670^{14,15}$. While the SS10 epitope was not reported to overlap with VZV gB $\beta 23$, HSV-1 gB residues $640-670$ are located in the region corresponding to $\mathrm{VZV} \mathrm{gB} \beta 30$, which is conserved in herpesvirus $\mathrm{gB}$ orthologues (Figs. 7 and 8). Neutralizing Ab epitopes have been mapped to five antigenic domains (AD) of HCMV gB ${ }^{4,16-18}$. Of these, DIV contains $\mathrm{AD}-1$ and $\mathrm{AD}-2$ (within the first $80 \mathrm{~N}$ terminal residues), DII contains $\mathrm{AD}-4$, DI contains $\mathrm{AD}-5$, and $\mathrm{AD}-3$ is at the C-terminus of full-length $\mathrm{gB}$. For HCMV, AD-1 is an immunodominant region for neutralizing antibodies ${ }^{51}$. Thus, these studies further support the role of DIV in gB-mediated membrane fusion initiation for both alpha- and betaherpesviruses.

In summary, this study has demonstrated the power of a cryoEM-based structure-function approach that solves the structure of a fusogenic viral glycoprotein recovered from infected cells in complex with a neutralizing antibody, thereby providing essential information to guide the biological discovery of glycoprotein residues critical for fusion function. These data provide compelling evidence that a domain independent of the $\mathrm{gB}$ fusion loops is required for herpesvirus fusion and has the potential to be utilized as a novel target for antiviral therapies.

\section{Methods}

Reagents and resources. All reagents, consumables, and resources, including supplier information where applicable, are provided in Supplementary Table 5.

Cells lines. All cell lines were propagated at $37^{\circ} \mathrm{C}$ in a humidified atmosphere with $5 \% \mathrm{CO}_{2}$. MeWo cells (HTB-65; ATCC) were propagated in minimal essential medium (Corning Cellgro) supplemented with 10\% fetal bovine serum (FBS; Invitrogen), nonessential amino acids ( $100 \mu \mathrm{M}$; Corning Cellgro), antibiotics (penicillin, $100 \mathrm{U} / \mathrm{ml}$; streptomycin, $100 \mu \mathrm{g} / \mathrm{ml}$; Invitrogen), and the antifungal agent amphotericin B (Invitrogen). CHO-DSP1 cells previously derived from the Chinese hamster ovary (CHO) K1 cell line (CCL-61; ATCC) and express the dual split protein (DSP1) R8(1-8) ${ }^{32}$ were propagated using F-12K nutrient mixture with Kaighn's modification (Invitrogen) supplemented with 10\% FBS and antibiotics (penicillin, $100 \mathrm{U} / \mathrm{ml}$; streptomycin, $100 \mu \mathrm{g} / \mathrm{ml}$; Invitrogen) and maintained under puromycin selection $(8 \mu \mathrm{g} / \mathrm{ml}$; Invitrogen). Mel-DSP2 cells previously derived from the MeWo cell line and express the DSP2 R8(9-11) 32 were propagated as for MeWo cells but under puromycin selection $(5 \mu \mathrm{g} / \mathrm{ml})$.

Viruses. The VZV parental Oka strain was originally cloned into a bacterial artificial chromosome (BAC) and designated pPOKA-BAC-DX ${ }^{52}$. The recombinant virus pOka-TK-GFP (pOka-rTK) was generated in a previous study ${ }^{36}$. All recombinant pOka-TK-GFP VZV mutants were derived from the self-excisable BAC, pPOKA-TK-GFP-BAC-DX $\triangle$ ORF31 ${ }^{36}$. The gB-KAN cassette ${ }^{53}$ was digested with BstZ171 and NaeI. The 4056-bp fragment was gel-purified and used to transform electrocompetent GS1783 Escherichia coli carrying the pPOKA-TK-GFP $\triangle \mathrm{ORF} 31 \mathrm{BAC}$. After red recombination, the pPOKA-TK-GFP BAC was purified using a large-construct purification kit (Qiagen). BACs were digested with Hind III to verify that spurious recombination had not occurred, and successful incorporation of ORF31 mutations were verified by sequencing the BAC directly. To generate BAC-derived VZV, $10^{6} \mathrm{MeW}$ cells seeded in six-well plates (Nunc) $24 \mathrm{~h}$ previously were transfected with $4 \mu \mathrm{g}$ of the pPOKA BACs using Lipofectamine 2000 (Invitrogen) following the manufacturer's instructions. Recombinant VZV was typically recovered at 5-10 days post-transfection. All virus stocks, pOka and gB DIV mutants, were sequenced to verify that the expected ORF31[gB] sequence was present. Briefly, DNA was extracted from infected cells using proteinase $\mathrm{K}$ and phenol/chloroform (Invitrogen). VZV ORF31 was amplified by PCR with KOD Extreme $^{\text {Tw }}$ (EMD Millipore) following the manufacturer's instructions using the oligonucleotides [31]F56625-56645/[31]R59697-59717. The PCR products were gel purified and sequenced by Sanger sequencing.

Construction of VZV pOka-TK-GFP-gB-TEVV5. For the purification of gB from $\mathrm{VZV}$-infected cells, a green fluorescent protein (GFP) expressing virus was generated that produced $\mathrm{gB}$ with a tag containing the tobacco etch virus protease cleavage site and a V5 epitope (TEVV5). A gB-Kan-TEVV5 shuttle vector was generated in a three-step cloning procedure. First, a gB-Kan-V5 vector was generated by amplifying two fragments from the gB-Kan vector ${ }^{53}$ using AccuPrime ${ }^{\text {th }}$ $P f x$ (Invitrogen) with oligonucleotides gB-AgeI/gB931 and gB-V5/M13R, purified using a QIAquick gel purification kit (QIAGEN) following the manufacturer's instructions, and ligated into the AgeI and SpeI site of the gB-Kan vector. Secondly two fragments were amplified from gB-Kan-V5 using AccuPrime ${ }^{\mathrm{rm}} \mathrm{Pf} x$ with oligonucleotides gB-AgeI/gB_cterm_Sprotein and gB_link_TEV_link/M13R, gel purified and ligated into the AgeI/SpeI site of gB-Kan. The final step deleted the Stag from the gB-Kan-STEVV5 to generate the gB-Kan-TEVV5 shuttle vector. Two fragments were amplified from gB-Kan-STEVV5 using AccuPrime ${ }^{\text {ru }}$ Pf $x$ with oligonucleotides gB-AgeI/ $\Delta$ S-tag-sense and $\Delta$ S-tag-antisense/M13R, gel purified, and ligated into the AgeI and SpeI site of the gB-Kan vector. The gB-Kan-TEVV5 shuttle vector was used to reconstitute ORF31-TEVV5 into the pOka-TK-GFP$\triangle$ ORF31 BAC to generate pPOKA-TK-GFP-gB-TEVV5 and recovery of pOka-TK GFP-gB-TEVV5 virus was performed as described in the 'Viruses' section.

Construction of the VZV pOka-TK-GFP-gB-TEVV5 gB DIV mutants. Site directed mutagenesis was performed using the pCAGGs-VZV gB (pCAGGs-gB) vector for template to generate DNA fragments using AccuPrime ${ }^{\text {tx }} P f x$. For the single alanine substitutions at S589A, R592A, and I594A, DNA fragments were amplified using the oligonucleotide combinations pCAGGs-gB-XmaI-sense/ S589A-antisense (S589A), S589A-sense/pCAGGs-gB-AgeI-antisense, pCAGGs-gB XmaI-sense/R592A-antisense (R592A), pCAGGs-gB-XmaI-sense/I594A-antisense (I594A), and pCAGGs-gB-3617-sense/pCAGGs-gB-AgeI-antisense. For the combined alanine substitutions at S589A/R592A/I594A $\left({ }^{589} \mathrm{AAA}^{594}\right)$, DNA fragments were amplified using the oligonucleotides pCAGGs-gB-XmaI-sense/589AAA594antisense and 589AAA594-sense/pCAGGs-gB-AgeI-antisense. For alanine substitutions at Q596, N597, and ${ }^{596} \mathrm{QN}^{597}$, DNA fragments were amplified using the oligonucleotide combinations pCAGGs-gB-XmaI-sense/Q596A-antisense (Q596A), pCAGGs-gB-3623-sense/pCAGGs-gB-AgeI-antisense, pCAGGs-gBXmaI-sense/pCAGGs-gB-3622-antisense, N597A-sense/pCAGGs-gB-AgeI-antisense (N597A), pCAGGs-gB-XmaI-sense/Q596A-antisense, and N597A-sense/ pCAGGs-gB-AgeI-antisense $\left({ }^{596} \mathrm{QN}^{597}\right)$. All DNA fragments were gel purified and digested with the appropriate restriction enzymes for ligation into the XmaI/AgeI site of pCAGGs-gB. To generate the ${ }^{592} \mathrm{~A} /{ }^{596} \mathrm{QN}^{597}$ substitutions the pCAGGs-gBR592A was used as template to amplify DNA fragments using oligonucleotides pCAGGs-gB-XmaI-sense/R592A-Q596A-antisense and N597A-sense/pCAGGsgB-AgeI-antisense. The two DNA fragments were gel purified and digested with the appropriate restriction enzymes for ligation into the $\mathrm{XmaI} / \mathrm{AgeI}$ site of pCAGGs-gB. For the alanine substitution Y667A, E670A and ${ }^{667} \mathrm{~A} / \mathrm{A}^{670}$, DNA fragments were amplified using the oligonucleotide combinations pCAGGs-gBMluI-sense/Y667A-antisense (Y667A), E670A-sense/pCAGGs-gB-AgeI-antisense (E670A), pCAGGs-gB-MluI-sense/Y667-antisense and V668-sense/pCAGGs-gBAgeI-antisense. All DNA fragments were gel purified and digested with the appropriate restriction enzymes for ligation into the MluI/AgeI site of pCAGGs-gB To generate the ${ }^{592} \mathrm{~A} /{ }^{596} \mathrm{QN}^{597} /{ }^{667} \mathrm{~A} / \mathrm{A}^{670}$ substitutions the pCAGGs-gB-592A/ 596AA597 was used as template to amplify DNA fragments using oligonucleotides pCAGGs-gB-MluI-sense/Y667A-antisense (Y667A) and E670A-sense/pCAGGsgB-AgeI-antisense (E670A). All the pCAGGs vectors were sequenced to verify that only the specific mutations were incorporated and spurious mutations from the PCR had not been incorporated.

The ${ }^{589} \mathrm{AAA}^{594},{ }^{596} \mathrm{AA}^{597},{ }^{592} \mathrm{~A} /{ }^{596} \mathrm{AA}^{597}$, and ${ }^{592} \mathrm{~A} /{ }^{596} \mathrm{AA}^{597} /{ }^{667} \mathrm{~A} / \mathrm{A}^{670}$ combined and single mutations were transferred into the gB-Kan-TEVV5 shuttle vector by restriction digest of the pCAGGS-gB mutant vectors and cloned into the NdeI/AgeI site of the gB-Kan-TEVV5. All the gB-Kan-TEVV5 vectors were sequenced to verify that only the specific mutations were incorporated and spurious mutations from the PCR had not been incorporated. The gB-Kan-TEVV5 shuttle vectors were used to reconstitute ORF31-TEVV5 DIV mutants into the pOka-TK-GFP- $\triangle$ ORF31 BAC to generate pPOKA-TK-GFP-gB-TEVV5 DIV mutant BACs and recovery of pOka-TK-GFP-gB-TEVV5 DIV mutant viruses was performed as described in the 'Viruses' section. Critically, sequencing of virus stocks confirmed that none of the gB $\beta 23$ mutants had unexpected nucleic acid substitutions in ORF31 (Supplementary Fig. 6).

Purification of native full-length gB from VZV-infected cells. MeWo cells were infected with the pOka-gB-TEVV5 virus and replication was allowed to proceed until cytopathic effect was observed across $90 \%$ of the cell monolayer. Infected cells 
were scrapped into ice cold PBS and pelleted at 424 RCF for 5 mins. Cells were lysed in glycoprotein extraction buffer (0.1 M Tris-base[pH7.2], $0.1 \mathrm{M} \mathrm{NaCl}, 5 \mathrm{mM}$ $\mathrm{KCL}, 1 \mathrm{mM} \mathrm{CaCl} 2,0.5 \mathrm{mM} \mathrm{MgCl}_{2}, 1 \%$ sodium deoxycholate, and $1 \% \mathrm{NP} 40$ ) plus an EDTA-free protease inhibitor cocktail (Roche, CA, USA) ${ }^{54}$. Cell lysates were clarified at 3000 RCF for 10 mins and $5 \mathrm{ml}$ of clarified lysate was incubated with $250 \mu \mathrm{l}$ of anti-V5 agarose beads (Sigma) for $2 \mathrm{~h}$ at room temperature. The beads were washed extensively in PBS $+0.1 \%$ Triton and a final wash with PBS then incubated with PBS containing TEV protease $(55 \mu \mathrm{g} / \mathrm{ml})$ for $20 \mathrm{~h}+4^{\circ} \mathrm{C}$. The TEV cleaved $\mathrm{gB}$ was eluted from the beads with TBS $\mathrm{pH} 7.4$ containing $1 \mathrm{mg} / \mathrm{ml}$ lauroylsarcosine (Sigma) and $1 \mathrm{mg} / \mathrm{ml}$ Amphipol 8-35 (Anatrace). Buffer exchange into TBS pH7.4 and $1 \mathrm{mg} / \mathrm{ml}$ Amphipol 8-35 using Amicon ${ }^{\circledR}$ Ultra-4 centrifugation filters with a $100 \mathrm{kDa}$ cutoff (Millipore). The concentration of Amphipol 8-35 was brought up to $35 \mathrm{mg} / \mathrm{ml}$ and incubated at room temperature for $4 \mathrm{~h}$ then BioBeads $^{\text {mm }}$ SM-2 (Bio-Rad) were added and incubated for $16 \mathrm{~h}$ at $4{ }^{\circ} \mathrm{C}$. The purified native, full-length $\mathrm{gB}$ was resolved on either Native PAGE or denaturing SDS-PAGE and either stained with Coomassie (Native PAGE) or Gel Code Blue (SDS-PAGE) following the manufacturer's instructions. To determine that the purified protein was native, full-length $\mathrm{gB}$ western blot was performed by transferring proteins to Immobilon-P membranes (Millipore Biosciences, Temecula, CA) and blocked with 5\% BSA. The mouse mAb SG2, the human mAb 93k and a rabbit polyclonal antiserum, 746-868, which recognizes the peptide sequence ${ }^{833}$ PEGMDPFAEKPNAT ${ }^{846}$ in the cytoplasmic region of pOka $\mathrm{gB}^{33}$, were used to detect $\mathrm{gB}$. Horse radish peroxidase conjugated antibodies that detect either mouse, human, or rabbit IgG (GE Healthcare Bio-Sciences Corp., Piscataway, NJ) were used and HRP activity detected using ECL plus (GE Healthcare Bio-Sciences Corp., Piscataway, NJ). The native, full-length $\mathrm{gB}$ was further purified on a Superose- 6 column (GE Healthcare Life Sciences) into TBS pH7.4 to remove aggregates.

Isolation of mAb 93k and preparation of 93k Fab. B-lymphocytes from a VZV immune individual were used to generate triomas that secrete antibodies that cross reacted with VZV gB and had neutralizing activity against 11 clinical isolates ${ }^{55}$. Triomas that secreted $\mathrm{gB}$ reactive antibodies were subcloned by limiting dilution resulting in clone 93kA9. The 93k Fab coding sequences for the variable heavy $(\mathrm{VH})$ chain and variable light $(\mathrm{VL})$ were subsequently sequenced and cloned into the pRS5a mammalian expression vector (Novartis AG, Basel, Switzerland), which expresses a generic constant region of IgG1 heavy chain (HC) and light chain (LC). The complete heavy chain has a cleavable double strep tag at the C-terminus. Fab fragments were generated from $\mathrm{mAb} 93 \mathrm{k}$ then purified by affinity and sizeexclusion column.

\section{Preparation of mAb 93k Fab fragments bound to the native full-length VZV} gB. Native full-length VZV gB was purified from infected cells as described in the 'Purification of native full-length gB from VZV-infected cells' section except the $\mathrm{mAb}$ 93k Fab fragments were added in molar excess immediately after the TBS pH7.4 and $1 \mathrm{mg} / \mathrm{ml}$ Amphipol 8-35 buffer exchange. The native full-length gB plus Fab fragments were incubated overnight at $+4^{\circ} \mathrm{C}$ on a rotary mixer. The complexes were concentrated using Amicon Ultra $10 \mathrm{kDa}$ filter units following the manufacturer's instructions. The concentration of Amphipol 8-35 was brought up to $35 \mathrm{mg} / \mathrm{ml}$ and incubated at room temperature for $4 \mathrm{~h}$ then Bio-Beads ${ }^{\mathrm{st}}$ SM-2 (BioRad) were added and incubated for $16 \mathrm{~h}$ at $4{ }^{\circ} \mathrm{C}$. The purified native, full-length gB-Fab complexes were evaluated by Native PAGE and purified on a Superose- 6 column (GE Healthcare Life Sciences) into TBS pH7.4 to remove aggregates.

Grid freezing. Lacey carbon copper 400 mesh grids with an ultrathin layer of carbon or Quantifoil R 1.2/1.3 gold 300 mesh grids were used for specimen freezing. EM grids were glow discharged for $25 \mathrm{~s}$. To each grid, $3 \mu \mathrm{l}$ of purified protein was dispensed and immediately plunge frozen into liquid ethane using Leica EM GP. Optimum chamber humidity and blotting times were determined empirically for each sample and ranged from 95 to $99 \%$ and $1.8-2.5 \mathrm{~s}$

Cryo-EM data collection. Micrographs for native, full-length VZV gB in complex with $93 \mathrm{k}$ Fab fragments were captured on a $300-\mathrm{kV}$ Titan Krios (FEI) controlled by SerialEM ${ }^{56}$ to automate the data collection procedure. Movie data (11,283 total stacks) were captured with a Gatan K2 Summit $(5 \mu \mathrm{m} /$ pixel) in counted mode with a dose rate of $\sim 1.335 \mathrm{e}^{-} / \AA^{2} / \mathrm{s}$ per frame and 200 millisecond exposure time per frame and 12-s total exposure time at a nominal magnification of $\times 130,000$ and a pixel size of $1.06 \AA /$ pixel on the specimen. The defocus range was $1.5-2.0 \mu \mathrm{m}$.

Map reconstruction of full-length VZV gB in complex with mAb 93k Fab. The motion correction and damage compensation for all movie-mode data were performed using MotionCor ${ }^{27}$. CTFFIND4 was used to estimate the contrast transfer function parameters ${ }^{58}$. Initially, the first 100 micrographs were selected to box out particle images using EMAN2's e2boxer.py ${ }^{59}$, followed by Relion's $\mathrm{s}^{60,61} 2 \mathrm{D}$ classification which generated a set of $2 \mathrm{D}$ class averages. The good $2 \mathrm{D}$ class averages were selected as templates to box out the particle images from all micrographs using Relion's auto-picking. EMAN2's e2initialmodel.py ${ }^{59}$ or Relion's 3D initial model was utilized to build the initial model. A couple of Relion's 2D classifications were first performed to remove junk, and the good classes were selected from 3D classification to do the final 3D auto-refine, for which 856,068 particles were selected. The C3 symmetry was imposed during the 3D auto-refine of Relion.

Structure and visualization of the VZV gB-93k complex. The resolution of the cryo-EM map and model of gB-93k was determined using Fourier shell correlation overall resolution estimate ${ }^{62}$. A protomer model was built by fitting our X-ray structure (PDB 6VLK; unpublished) and the 93k VH and VL chains were built de novo. The gB-93k structure models were generated and refined using $\operatorname{Coot}^{63}$ and Phenix ${ }^{64-67}$. ResMap was used to calculate local resolution variation ${ }^{34}$. A newly developed Q scoring tool was applied to calculate feature resolvability ${ }^{35}$. Interactions between amino acids were calculated using the Find Contacts tool in UCSF Chimera 1.13.1 using the default settings ${ }^{68}$. Surface electrostatic potential was calculated using APBS (Adaptive Poisson-Boltzmann Solver) ${ }^{69}$. All images and movies were generated using the Animation tool in UCSF Chimera 1.13.1.

Cell-free VZV neutralization assay. Cell-free VZV stocks were prepared as described ${ }^{70}$. MeWo cells in $100 \mathrm{~mm}$ culture dishes infected with pOka-TK-GFP were washed with cold PBS then incubated at room temperature with PBS + 0.1\% EDTA The cells were dislodged by pipetting, centrifuged at 424 RCF for 5 mins then resuspended in PSGC buffer (PBS + $145 \mathrm{M}$ sucrose $+6 \mathrm{mM} \mathrm{L}(+)$-glutamic acid + $10 \% \mathrm{FBS}$ ). The resuspended cells were transferred to a Dounce homogenizer $\left(\right.$ KONTES $\left.^{\circ}\right)$ and disrupted with 15 strokes of pestle A and 15 strokes of pestle B. The resulting homogenate was centrifuged at $3000 \mathrm{RCF}$ to removed cell debris and the supernatant was stored in $1 \mathrm{ml}$ aliquots under liquid nitrogen. The titers of cell-free VZV were typically in the $3.5 \log _{10} / \mathrm{ml}$ range. To test antibodies for VZV neutralization capabilities, $100 \mathrm{pfu}$ of cell-free pOka-TK-GFP was incubated with $10 \mu \mathrm{g}$ antibody (93k, SG2 or 206) at room temperature for 30 mins. MeWo cells seeded $24 \mathrm{~h}$ previously at $1 \times 10^{5} / \mathrm{cm}^{2}$ in 12 -well plates were inoculated with the pOka-TKGFP/antibody mixtures and incubated for $24 \mathrm{~h}$. The media was changed, and the plates were incubated for a further $72 \mathrm{~h}$ then fixed with $4 \%$ paraformaldehyde. Plaques were detected by immunohistochemical staining using an anti-VZV mixed mouse $\mathrm{mAb}$ followed by detection with a streptavidin conjugated goat anti-mouse IgG (Jackson ImmunoResearch Laboratories, Inc., West Grove, PA) and alkaline phosphatase conjugated avidin (Jackson ImmunoResearch Laboratories, Inc., West Grove, PA). Enzyme activity was detected using a fast red substrate (0.1MTris - $\mathrm{pH}$ 8.0, $5 \mu \mathrm{M}$ Naphthol AS-Mx phosphate (Sigma), $80 \mu \mathrm{M}$ Fast Red TR (Sigma)).

\section{Quantitation of cell surface gB for the DIV mutants. CHO-DSP1 cells $(8 \times$} $10^{5}$ cells/well) in six-well plates were transfected with $5 \mu \mathrm{g}$ WT or mutant pCAGGS$\mathrm{gB}$ expression vectors. Cells were dislodged at $24 \mathrm{~h}$ post transfection using an enzyme-free cell dissociation buffer (Life Technologies, Grand Island, NY), washed with PBS then fixed with $1 \%$ paraformaldehyde. The fixed cells were washed with PBS then resuspended in FACS staining buffer (DPBS (Dulbecco's PhosphateBuffered Saline; Cellgro, Manassas, VA) with 0.2\% IgG-free BSA (Jackson ImmunoResearch, West Grove, PA) and 0.1\% NaN3 (Sigma Aldrich, St. Louis, MO)) for cell surface staining with anti-VZV gB mAb SG2-2E6 or 93k. A donkey anti-mouse IgG-Alexa Fluor 555 antibody (SG2-2E6) or goat anti-human IgG-Alexa Fluor 488 antibody (93k) (Life Technologies, Grand Island, NY) was used to detect bound anti-VZV gB mAb. Total $\mathrm{gB}$ expression was determined by using the same staining protocol except cells were permeabilized using Cytofix/Cytoperm (BD Biosciences, San Jose, CA) before adding the primary antibody and during the staining procedure. Stained cells were analyzed using a FACSCalibur with CellQuest Pro (BD Biosciences, San Jose, CA). FlowJo (TreeStar, Ashland, OR) was used to determine the quantity of total and cell surface gB on the transfected cells. The quantities for $\mathrm{gB}$ mutants were normalized to $\mathrm{WT} \mathrm{gB}$, which was set at $100 \%$. Experiments were performed with at least two gB mutant clones, each tested in duplicate.

VZV stable reporter fusion assay. The stable reporter fusion assay for the VZV glycoproteins $\mathrm{gB} / \mathrm{gH}$-gL has been reported previously 32 but was adapted for use with a 96-well plate format. CHO-DSP1 cells seeded at $8 \times 10^{5}$ per well in six-well plates $20 \mathrm{~h}$ previously were transfected with $1.6 \mu \mathrm{g}$ each of pCAGGs-gB, pME18s$\mathrm{gH}[\mathrm{TL}]$, and pCDNA-gL plasmids with Lipofectamine 2000 following the manufacturer's instructions. At $6 \mathrm{~h}$ post transfection, the transfected CHO-DSP1 cells were trypsinized, collected by centrifugation at $424 \mathrm{RCF}$, and resuspended in $1 \mathrm{ml}$ of medium, of which $250 \mu \mathrm{l}$ of cells were mixed with $0.75 \mathrm{ml}$ of Mel-DSP2 cells at $10^{6}$ cells $/ \mathrm{ml}$. To test fusion inhibition properties of mAbs, 93k, SG2 and 206, $10 \mu \mathrm{g}$ of antibody was added to the cell mixtures. The cells were mixed by inversion and $75 \mu \mathrm{l}$ of the suspension was dispensed to at least triplicate wells of 96-well blacked sided optical bottom plates culture plates (Thermo Scientific). At $40 \mathrm{~h}$ post seeding, $50 \mu \mathrm{l}$ membrane permeable coelenterazine- $\mathrm{H}(5 \mu \mathrm{M}$, Nanolight Technology) substrate for five mins at room temperature. Fusion was quantified by measuring luminescence using a Synergy H1 Multi-mode reader (Biotek). A minimum of two clones were tested in duplicate experiments.

Immunofluorescence staining of MeWo cells. To each well of a 12-well plate (Nunclon $^{\text {tw }}$ Delta Surface; Thermo Scientific) a sterile $18 \mathrm{~mm}$ coverslip (Fisher Scientific) was placed and $2 \mathrm{ml}$ of MeWo cells at $2 \times 10^{5} / \mathrm{ml}$ was dispensed and incubated overnight. MeWo cells were transfected with 2 ug of pPOKA-TK-GFP BACs carrying gB mutants using Lipofectamine 2000 following the manufacturer's 
instructions. At $72 \mathrm{~h}$ post transfection, the media was aspirated, the coverslips washed with PBS and fixed with $4 \%$ paraformaldehyde for 10 mins. Immunofluorescence was performed by blocking the cells with PBS $+10 \%$ normal donkey serum (NDS) $+0.1 \%$ Triton X-100 then adding a mouse mAb to the immediate early protein IE62 in PBS $+1 \%$ NDS $+0.1 \%$ Triton X-100. The anti-IE62 mAb was detected with the donkey anti-mouse IgG-Alexa Fluor 555 and nuclei were stained with Hoechst 33342 in PBS $+1 \%$ NDS $+0.1 \%$ Triton X-100. Coverslips were mounted on glass slides (Selectfrost; Fisher Scientific) using Fluoromount-G (SouthernBiotech) and a minimum of five images were captured for each transfection using a Keyence fluorescence microscope using a $\times 20$ objective.

Quantitation of plaque sizes for the VZV gB DIV mutants. MeWo cells were seeded at $10^{6}$ cells/well $24 \mathrm{~h}$ prior to inoculation with $50 \mathrm{pfu}$ of either wild type pOka or gB DIV mutants. Each well of the six-well plate was fixed at 4 days post inoculation with $4 \%$ formaldehyde and stained by immunohistochemistry. Images of stained plaques $(n=40)$ were digitally captured, the stained plaque was outlined, and the area $\left(\mathrm{mm}^{2}\right)$ was calculated using ImageJ (National Institute of Mental Health). Statistical analyses were performed using Prism (GraphPad Software)

Immunoprecipitation of VZV gB DIV mutants. CHO-DSP1 cells seeded in sixwell plates were transfected with $5 \mu \mathrm{g} /$ well of pCAGGS-gB vectors carrying the DIV mutations using Lipofectamine 2000 following the manufacturer's instructions. At $24 \mathrm{~h}$ post transfection cells were lysed with glycoprotein lysis buffer, the same buffer used for the purification of native, full-length VZV gB, and snap frozen in liquid nitrogen and stored at $-20^{\circ} \mathrm{C}$. The SG2 or $93 \mathrm{k}$ mAbs were cross-linked to immobilized protein A (Pierce, Rockford, IL) ${ }^{71}$. Each $20 \mu \mathrm{g}$ of mAb was incubated with $30 \mu \mathrm{l}$ protein A beads for $1 \mathrm{~h}$ at room temperature on a rotary mixer. The beads were washed with DPBS then mAbs were cross-linked to the beads with $0.2 \mathrm{M}$ sodium borate [pH9.0] and $20 \mathrm{mM}$ DMP for 30 mins. The cross-linking reaction was quenched with $0.2 \mathrm{M} \mathrm{NaCl}$ and $0.2 \mathrm{M}$ ethanolamine [pH 8.0] for $2 \mathrm{~h}$ at room temperature. The cross-linked beads were washed with DPBS. Lysates from the pCAGGs-gB transfected CHO-DSP1 cells were divided equally and incubated overnight at $+4{ }^{\circ} \mathrm{C}$ with either the SG2 or 93k cross-linked beads. The beads were washed extensively with DPBS $+0.1 \%$ Triton X-100 and a final wash of DPBS to remove the Triton X-100. Bound proteins were eluted into sodium dodecyl sulfate (SDS) sample buffer (Bio-Rad) containing 5\% 2-mercaptoethanl (Sigma) by incubating the beads at $100^{\circ} \mathrm{C}$ for $5 \mathrm{~min}$. Denatured samples were resolved on SDSpolyacrylamide gel electrophoresis precast gels (Bio-Rad, Hercules, CA) and western blot was performed using the 746-868 rabbit poly clonal IgG.

Immunoprecipitation of VZV gB DIV mutants in complex with gH-gL. CHODSP1 cells were transfected as described in the previous section with pCAGGS-gB vectors carrying the DIV mutations, pME18s-gH[V5] and pCDNA3.1-gL (1.6 $\mu \mathrm{g}$ of each vector). At $24 \mathrm{~h}$ post transfection cells were lysed with glycoprotein lysis buffer and snap frozen in liquid nitrogen and stored at $-20^{\circ} \mathrm{C}$. The $\mathrm{gB} / \mathrm{gH}$-gL complexes were immunoprecipitated with anti-V5 agarose (Sigma). Wash steps, protein elution, and SDS-PAGE were performed as outlined in the previous section. Western blots were performed using either mouse anti-V5 tag (Bio-Rad), 746-868 rabbit poly clonal IgG, or $\mathrm{mAb} 93 \mathrm{k}$.

Statistics and reproducibility. All quantitative data were analyzed with two-way ANOVA to determine statistical significance using Prism (GraphPad Software). All statistical analyses are presented in the Source Data file. Images of gB-93k class averages in Fig. 2a are representative of 23 classifications. Confocal micrographs in Figs. $5 \mathrm{c}$ and $6 \mathrm{c}$ are representative images of $n=10$ from two independent experiments. Images of gels and western blots in Figs. 1a, 5e, f, and 6e, f and Supplementary Figs. $2 a, b$ and 7 are representative of at least two independent experiments.

Reporting summary. Further information on research design is available in the Nature Research Reporting Summary linked to this article.

\section{Data availability}

Maps and models have been deposited in the Electron Microscopy Data Bank with accession code EMD-21247 and the Protein Data Bank with accession code 6VN1. Source data are provided with this paper. All primary data will be provided by the corresponding author upon reasonable request. Source data are provided with this paper.

Received: 4 March 2020; Accepted: 21 July 2020;

Published online: 18 August 2020

\section{References}

1. Pellett, P. E. \& Roizman, B. In Fields Virology, Vol. 2 (eds Bernard N. Fields, David M. Knipe, \& Peter M. Howley) 1802-1822 (Wolters Kluwer Health/ Lippincott Williams \& Wilkins, 2013).
2. Backovic, M., Longnecker, R. \& Jardetzky, T. S. Structure of a trimeric variant of the Epstein-Barr virus glycoprotein B. Proc. Natl Acad. Sci. USA 106, 2880-2885 (2009)

3. Burke, H. G. \& Heldwein, E. E. Crystal structure of the human cytomegalovirus glycoprotein B. PLoS Pathog. 11, e1005227 (2015).

4. Chandramouli, S. et al. Structure of HCMV glycoprotein B in the postfusion conformation bound to a neutralizing human antibody. Nat. Commun. 6 , 8176 (2015)

5. Heldwein, E. E. et al. Crystal structure of glycoprotein B from herpes simplex virus 1. Science 313, 217-220 (2006)

6. Roche, S., Bressanelli, S., Rey, F. A. \& Gaudin, Y. Crystal structure of the low-pH form of the vesicular stomatitis virus glycoprotein G. Science 313, 187-191 (2006)

7. Roche, S., Rey, F. A., Gaudin, Y. \& Bressanelli, S. Structure of the prefusion form of the vesicular stomatitis virus glycoprotein G. Science 315, 843-848 (2007).

8. Kadlec, J., Loureiro, S., Abrescia, N. G., Stuart, D. I. \& Jones, I. M. The postfusion structure of baculovirus gp64 supports a unified view of viral fusion machines. Nat. Struct. Mol. Biol. 15, 1024-1030 (2008).

9. Connolly, S. A., Jackson, J. O., Jardetzky, T. S. \& Longnecker, R. Fusing structure and function: a structural view of the herpesvirus entry machinery. Nat. Rev. Microbiol. 9, 369-381 (2011).

10. Zeev-Ben-Mordehai, T. et al. Two distinct trimeric conformations of natively membrane-anchored full-length herpes simplex virus 1 glycoprotein B. Proc. Natl Acad. Sci. USA 113, 4176-4181 (2016).

11. Maurer, U. E. et al. The structure of herpesvirus fusion glycoprotein B-bilayer complex reveals the protein-membrane and lateral protein-protein interaction. Structure 21, 1396-1405 (2013).

12. Cooper, R. S., Georgieva, E. R., Borbat, P. P., Freed, J. H. \& Heldwein, E. E. Structural basis for membrane anchoring and fusion regulation of the herpes simplex virus fusogen gB. Nat. Struct. Mol. Biol. 25, 416-424 (2018).

13. $\mathrm{Si}, \mathrm{Z}$. et al. Different functional states of fusion protein $\mathrm{gB}$ revealed on human cytomegalovirus by cryo electron tomography with Volta phase plate. PLoS Pathog. 14, e1007452 (2018).

14. Cairns, T. M. et al. Capturing the herpes simplex virus core fusion complex (gB-gH/gL) in an acidic environment. J. Virol. 85, 6175-6184 (2011).

15. Bender, F. C. et al. Antigenic and mutational analyses of herpes simplex virus glycoprotein B reveal four functional regions. J. Virol. 81, 3827-3841 (2007).

16. Aulitzky, W. E. et al. Human monoclonal antibodies neutralizing cytomegalovirus (CMV) for prophylaxis of CMV disease: report of a phase I trial in bone marrow transplant recipients. J. Infect. Dis. 163, 1344-1347 (1991).

17. Ohlin, M., Sundqvist, V. A., Mach, M., Wahren, B. \& Borrebaeck, C. A. Fine specificity of the human immune response to the major neutralization epitopes expressed on cytomegalovirus gp58/116 (gB), as determined with human monoclonal antibodies. J. Virol. 67, 703-710 (1993).

18. Potzsch, S. et al. B cell repertoire analysis identifies new antigenic domains on glycoprotein B of human cytomegalovirus which are target of neutralizing antibodies. PLoS Pathog. 7, e1002172 (2011).

19. Meyer, H., Masuho, Y. \& Mach, M. The gp116 of the gp58/116 complex of human cytomegalovirus represents the amino-terminal part of the precursor molecule and contains a neutralizing epitope. J. Gen. Virol. 71, 2443-2450 (1990).

20. Spindler, N. et al. Structural basis for the recognition of human cytomegalovirus glycoprotein B by a neutralizing human antibody. PLoS Pathog. 10, e1004377 (2014).

21. Spindler, N. et al. Characterization of a discontinuous neutralizing epitope on glycoprotein B of human cytomegalovirus. J. Virol. 87, 8927-8939 (2013).

22. Cairns, T. M. et al. Mechanism of neutralization of herpes simplex virus by antibodies directed at the fusion domain of glycoprotein B. J. Virol. 88, $2677-2689$ (2014)

23. Li, X. et al. Two classes of protective antibodies against Pseudorabies virus variant glycoprotein B: Implications for vaccine design. PLoS Pathog. 13, e1006777 (2017).

24. Arvin, A. M. \& Gilden, D. In Fields Virology, Vol. 2 (eds Bernard N. Fields, David M. Knipe, \& Peter M. Howley) 2015-2184 (Wolters Kluwer Health/ Lippincott Williams \& Wilkins, 2013).

25. Weigle, K. A. \& Grose, C. Common expression of varicella-zoster viral glycoprotein antigens in vitro and in chickenpox and zoster vesicles. J. Infect. Dis. 148, 630-638 (1983).

26. Grose, C. \& Brunel, P. A. Varicella-zoster virus: isolation and propagation in human melanoma cells at 36 and 32 degrees C. Infect. Immun. 19, 199-203 (1978).

27. Gilden, D. \& Nagel, M. Varicella zoster virus in temporal arteries of patients with giant cell arteritis. J. Infect. Dis. 212, S37-S39 (2015).

28. Gilden, D. et al. Varicella zoster virus infection in granulomatous arteritis of the aorta. J. Infect. Dis. 213, 1866-1871 (2016).

29. Gilden, D. et al. Prevalence and distribution of VZV in temporal arteries of patients with giant cell arteritis. Neurology 84, 1948-1955 (2015).

30. Suenaga, T. et al. Myelin-associated glycoprotein mediates membrane fusion and entry of neurotropic herpesviruses. Proc. Natl Acad. Sci. USA 107, 866-871 (2010). 
31. Vleck, S. E. et al. Structure-function analysis of varicella-zoster virus glycoprotein $\mathrm{H}$ identifies domain-specific roles for fusion and skin tropism. Proc. Natl Acad. Sci. USA 108, 18412-18417 (2011).

32. Yang, E., Arvin, A. M. \& Oliver, S. L. Role for the alphaV integrin subunit in varicella-zoster virus-mediated fusion and infection. J. Virol. 90, 7567-7578 (2016).

33. Oliver, S. L. et al. Mutagenesis of varicella-zoster virus glycoprotein B: putative fusion loop residues are essential for viral replication, and the furin cleavage motif contributes to pathogenesis in skin tissue in vivo. J. Virol. 83, 7495-7506 (2009).

34. Kucukelbir, A., Sigworth, F. J. \& Tagare, H. D. Quantifying the local resolution of cryo-EM density maps. Nat. Methods 11, 63-65 (2014).

35. Pintilie, G. et al. Measurement of atom resolvability in cryo-EM maps with Qscores. Nat. Methods 17, 328-334 (2020).

36. Yang, E., Arvin, A. M. \& Oliver, S. L. The cytoplasmic domain of varicellazoster virus glycoprotein $\mathrm{H}$ regulates syncytia formation and skin pathogenesis. PLoS Pathog. 10, e1004173 (2014).

37. Vallbracht, M. et al. Structure-function dissection of the Pseudorabies virus glycoprotein B fusion loops. J. Virol. 92, e01203-17 (2017).

38. Atanasiu, D. et al. Bimolecular complementation defines functional regions of Herpes simplex virus $\mathrm{gB}$ that are involved with $\mathrm{gH} / \mathrm{gL}$ as a necessary step leading to cell fusion. J. Virol. 84, 3825-3834 (2010).

39. Avitabile, E., Forghieri, C. \& Campadelli-Fiume, G. Cross talk among the glycoproteins involved in herpes simplex virus entry and fusion: the interaction between $\mathrm{gB}$ and $\mathrm{gH} / \mathrm{gL}$ does not necessarily require $\mathrm{gD}$. J. Virol. 83, 10752-10760 (2009).

40. Backovic, M. \& Jardetzky, T. S. Class III viral membrane fusion proteins. Curr. Opin. Struct. Biol. 19, 189-196 (2009).

41. Gallagher, J. R. et al. Functional fluorescent protein insertions in herpes simplex virus gB report on $\mathrm{gB}$ conformation before and after execution of membrane fusion. PLoS Pathog. 10, e1004373 (2014).

42. Fontana, J. et al. The fusion loops of the initial prefusion conformation of Herpes Simplex Virus 1 fusion protein point toward the membrane. MBio 8 , e01268-17 (2017)

43. Li, Q., Turk, S. M. \& Hutt-Fletcher, L. M. The Epstein-Barr virus (EBV) BZLF2 gene product associates with the $\mathrm{gH}$ and gL homologs of EBV and carries an epitope critical to infection of B cells but not of epithelial cells. $J$. Virol. 69, 3987-3994 (1995).

44. Ryckman, B. J., Chase, M. C. \& Johnson, D. C. HCMV gH/gL/UL128-131 interferes with virus entry into epithelial cells: evidence for cell type-specific receptors. Proc. Natl Acad. Sci. USA 105, 14118-14123 (2008).

45. Wang, D. \& Shenk, T. Human cytomegalovirus virion protein complex required for epithelial and endothelial cell tropism. Proc. Natl Acad. Sci. USA 102, 18153-18158 (2005).

46. Satoh, T. et al. PILRalpha is a herpes simplex virus-1 entry coreceptor that associates with glycoprotein B. Cell 132, 935-944 (2008).

47. Soroceanu, L., Akhavan, A. \& Cobbs, C. S. Platelet-derived growth factoralpha receptor activation is required for human cytomegalovirus infection. Nature 455, 391-395 (2008).

48. $\mathrm{Wu}, \mathrm{Y}$. et al. Human cytomegalovirus glycoprotein complex $\mathrm{gH} / \mathrm{gL} / \mathrm{gO}$ uses PDGFR-alpha as a key for entry. PLoS Pathog. 13, e1006281 (2017).

49. Wang, H. B. et al. Neuropilin 1 is an entry factor that promotes EBV infection of nasopharyngeal epithelial cells. Nat. Commun. 6, 6240 (2015).

50. Suenaga, T. et al. Sialic acids on varicella-zoster virus glycoprotein B are required for cell-cell fusion. J. Biol. Chem. 290, 19833-19843 (2015).

51. Schoppel, K. et al. Antibodies specific for the antigenic domain 1 of glycoprotein B (gpUL55) of human cytomegalovirus bind to different substructures. Virology 216, 133-145 (1996).

52. Tischer, B. K. et al. A self-excisable infectious bacterial artificial chromosome clone of varicella-zoster virus allows analysis of the essential tegument protein encoded by ORF9. J. Virol. 81, 13200-13208 (2007).

53. Oliver, S. L. et al. An immunoreceptor tyrosine-based inhibition motif in varicella-zoster virus glycoprotein B regulates cell fusion and skin pathogenesis. Proc. Natl Acad. Sci. USA 110, 1911-1916 (2013).

54. Grose, C., Edwards, D. P., Weigle, K. A., Friedrichs, W. E. \& McGuire, W. L. Varicella-zoster virus-specific gp140: a highly immunogenic and disulfidelinked structural glycoprotein. Virology 132, 138-146 (1984).

55. Ostberg, L. \& Pursch, E. Human X (mouse X human) hybridomas stably producing human antibodies. Hybridoma 2, 361-367 (1983).

56. Mastronarde, D. N. Automated electron microscope tomography using robust prediction of specimen movements. J. Struct. Biol. 152, 36-51 (2005).

57. Zheng, S. Q. et al. MotionCor2: anisotropic correction of beam-induced motion for improved cryo-electron microscopy. Nat. Methods 14, 331-332 (2017).

58. Rohou, A. \& Grigorieff, N. CTFFIND4: fast and accurate defocus estimation from electron micrographs. J. Struct. Biol. 192, 216-221 (2015).

59. Tang, G. et al. EMAN2: an extensible image processing suite for electron microscopy. J. Struct. Biol. 157, 38-46 (2007).
60. Scheres, S. H. RELION: implementation of a Bayesian approach to cryo-EM structure determination. J. Struct. Biol. 180, 519-530 (2012).

61. Zivanov, J. et al. New tools for automated high-resolution cryo-EM structure determination in RELION-3. Elife 7, e42166 (2018).

62. Henderson, R. et al. Outcome of the first electron microscopy validation task force meeting. Structure 20, 205-214 (2012).

63. Emsley, P., Lohkamp, B., Scott, W. G. \& Cowtan, K. Features and development of Coot. Acta Crystallogr. D Biol. Crystallogr. 66, 486-501 (2010).

64. Adams, P. D. et al. PHENIX: a comprehensive Python-based system for macromolecular structure solution. Acta Crystallogr. D Biol. Crystallogr. 66, 213-221 (2010).

65. Williams, C. J. et al. MolProbity: more and better reference data for improved all-atom structure validation. Protein Sci. 27, 293-315 (2018).

66. Afonine, P. V. et al. New tools for the analysis and validation of cryo-EM maps and atomic models. Acta Crystallogr. D Struct. Biol. 74, 814-840 (2018).

67. Afonine, P. V. et al. Real-space refinement in PHENIX for cryo-EM and crystallography. Acta Crystallogr. D Struct. Biol. 74, 531-544 (2018).

68. Pettersen, E. F. et al. UCSF Chimera-a visualization system for exploratory research and analysis. J. Comput. Chem. 25, 1605-1612 (2004).

69. Jurrus, E. et al. Improvements to the APBS biomolecular solvation software suite. Protein Sci. 27, 112-128 (2018)

70. Kyratsous, C. A. \& Silverstein, S. J. Components of nuclear domain 10 bodies regulate varicella-zoster virus replication. J. Virol. 83, 4262-4274 (2009).

71. Harlow, E. \& Lane, D. Using Antibodies: A Laboratory Manual (Cold Spring Harbor Laboratory Press, 1999).

\section{Acknowledgements}

We thank the Stanford-SLAC Cryo-EM Facility, the Stanford Bio-X Interdisciplinary Initiatives Program, and NIH grants P41-GM103832, R01-GM079429, R01-AI102546, R37-AI20459, and S10-OD021600 for their valuable support. We thank the Roger Kornberg lab at Stanford University for providing essential reagents and cryo-EM access Molecular graphics and analyses performed with UCSF Chimera, developed by the Resource for Biocomputing, Visualization, and Informatics at the University of California, San Francisco, with support from NIH P41-GM103311.

\section{Author contributions}

S.L.O., Y.X., W.C., A.C., and A.M.A. wrote the paper. S.L.O., Y.X., and D.H.C. performed the experiments. S.L.O., Y.X., DH.C., S.H.R., G.D.P., and D.A.B. analyzed the data. S.L.O., Y.X., D.A.B., M.H.S., E.Y., and A.C. contributed reagents and materials. S.L.O., Y.X., DH C., D.A.B., A.C., W.C., and A.M.A. conceived and designed experiments. S.L.O., W.C., and A.M.A. jointly supervised research. All the authors discussed the data and contributed intellectually to the manuscript.

\section{Competing interests}

The authors declare no competing interests.

\section{Additional information}

Supplementary information is available for this paper at https://doi.org/10.1038/s41467 020-17911-0.

Correspondence and requests for materials should be addressed to S.L.O

Peer review information Nature Communications thanks the anonymous reviewers for their contribution to the peer review of this work. Peer reviewer reports are available.

Reprints and permission information is available at http://www.nature.com/reprints

Publisher's note Springer Nature remains neutral with regard to jurisdictional claims in published maps and institutional affiliations.

(i)

Open Access This article is licensed under a Creative Commons Attribution 4.0 International License, which permits use, sharing, adaptation, distribution and reproduction in any medium or format, as long as you give appropriate credit to the original author(s) and the source, provide a link to the Creative Commons license, and indicate if changes were made. The images or other third party material in this article are included in the article's Creative Commons license, unless indicated otherwise in a credit line to the material. If material is not included in the article's Creative Commons license and your intended use is not permitted by statutory regulation or exceeds the permitted use, you will need to obtain permission directly from the copyright holder. To view a copy of this license, visit http://creativecommons.org/ licenses/by/4.0/

(c) The Author(s) 2020 